\title{
Analysis of Immune Infiltration Pattern in Osteosarcoma and Its Clinical Significance
}

\section{Wenhua Yuan}

Department of Orthopedics,Xichang people's Hospital, xichang, sichuan https://orcid.org/0000-00019303-5832

\section{Yajun Deng}

Department of orthopedics, No.215 Hospital of Shanxi Nuclear Industry, xianyang, shanxi

\section{Enhui Ren}

Department of Orthopaedics, Xining First People's Hospital, Xining, Qinghai

\section{Guangzhi Zhang}

Department of orthopaedics, Lanzhou University Second Hospital, Lanzhou, Gansu

\section{Zuolong Wu}

Department of Orthopaedics, Lanzhou University Second Hospital, Lanzhou, Gansu

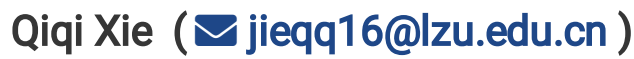

Affiliated Hospital of Qinghai University https://orcid.org/0000-0003-4099-5287

\section{Primary research}

Keywords: osteosarcoma, tumor microenvironment, tumor infiltrating immune cells, diagnostic markers, prognostic markers, targeted therapy

Posted Date: October 15th, 2020

DOI: https://doi.org/10.21203/rs.3.rs-91597/v1

License: (c) (1) This work is licensed under a Creative Commons Attribution 4.0 International License. Read Full License 


\section{Abstract}

Objective

Osteosarcoma (OS), the most common malignant bone tumor, is prone to early metastasis and recurrence, resulting in poor prognosis. The purpose of this study was to investigate gene expression in OS samples, and to study the relationship between tumor infiltrating immune cells (TIICS) in the tumor microenvironment and the occurrence, development, and prognosis of OS.

\section{Methods}

Integrated analysis was performed on the gene expression profile of OS samples in the Therapeutically Applicable Research to Generate Effective Treatments (TARGET) database, and normal muscle tissue samples in the Genotype-Tissue Expression (GTEx) database. Screening of differentially expressed genes in OS samples and healthy muscle tissue samples was performed using R software. Functional annotation and pathway enrichment analyses were performed, a protein-protein interaction network was constructed, and the Hub genes were screened. The infiltration differences of 22 TIICs in OS samples and normal muscle tissue samples were analyzed using CIBERSORT. ROC curve, and OS survival analyses were performed on the Hub genes and 22 TIICs. The correlation between Hub genes with prognostic value and the 22 TIICs was discussed.

Results

Ten Hub genes were screened. OS and normal muscle tissue samples were compared. Significant differences were observed in the infiltration of follicular helper T cells, resting NK cells, plasma cells, activated CD4 memory T cells, gamma delta T cells, monocytes, M0 macrophages and resting dendritic cells $(P<0.05)$. ROC curve analysis showed that the area under the curve (AUC) for the 10 hub genes was $0.9 \sim 1.0$, and for naive B cells (AUC=0.942), plasma cells (AUC=0.999), follicular $T$ helper cells (AUC=0.814), resting NK cells (AUC=0.864), monocytes ( $A U C=0.987)$, and M0 macrophages $(A U C=0.995)$. Kaplan-Meier curves revealed positive correlation between the expression levels of TNNI2, TTN, ACTN3, $T N N C 2, M Y L 3$, and survival of OS patients. Correlation analysis revealed close association between ACTN3, TNNI2, TNNC2, MYL3, TTN, and the infiltration of M0 macrophages and plasma cells.

\section{Conclusion}

Our findings provide a theoretical basis for further study of the molecular mechanisms underlying OS development and occurrence. It serves as a reference for the development of new diagnostics, identification of prognostic biomarkers, and targeted drug therapy for OS.

\section{Introduction}

Osteosarcoma (OS) is the most common primary malignant bone tumor, often occurring in children and adolescents [1]. Over the past three decades, although the development of surgical resection and 
neoadjuvant chemotherapy have significantly improved the prognosis of OS [2], the long-term survival rate of OS patients with recurrence or pre-existing metastasis is still less than $20 \%[3,4]$. Therefore, elucidating the mechanism of development and occurrence of OS, and finding effective diagnostic techniques, prognostic biomarkers and treatment targets, is of great importance. Tumor cells are located in the tumor microenvironment (TME), which is composed of stroma and tumor-infiltrating immune cells (TIICs). The TIICs and molecules exist in a dynamic state, and this movement is strongly associated with the development, occurrence, and prognosis of the tumor [5-7]. Heymann et al. [8] reported that OS cells control the recruitment and differentiation of TIICS, and establish a local immune tolerance environment conducive to tumor growth, drug resistance, and metastasis. Some studies have found that the gene expression level in OS was strongly associated with the occurrence, recurrence, and metastasis of these cells [3,9]. Scott et al. [10] have shown that the gene expression level in OS may be strongly associated with the infiltration of immune cells. However, there is a lack in the comprehensive understanding of the differentially expressed genes (DEGs) in OS, as well as TIICs in their TME.

Owing to the rapid development in gene expression microarray technology, big data mining analysis can be performed using bioinformatics methods [11]. CIBERSORT (https://cibersort.stanford.edu/), an analysis tool developed by Newman et al. [12], can perform large-scale analysis of RNA mixtures, and can accurately estimate the type of the 22 TIICs in their TME. Gene set variation analysis (GSVA), another method, is non-parametric and unsupervised. By transforming the gene data matrix of all samples into the gene set, which is composed of the matrix of each sample, each sample is enriched through the pathway, which helps to estimate the variation of gene set enrichment through the sample gene expression profile data set [13].

The Therapeutically Applicable Research to Generate Effective Treatments (TARGET) database (https://ocg.cancer.gov programs/target) is a new database, which tries to identify molecular changes in the occurrence and development of refractory childhood cancers, using a comprehensive genomic approach. The Genotype-Tissue Expression (GTEx) database provides transcriptome data for various normal human tissues [14]. In this study, the gene chip sequencing data of normal muscle tissue samples was downloaded from the GTEx database (control), and the OS gene chip sequencing data was downloaded from the TARGET database, for the first time. Through relevant bioinformatics methods, the gene chip data matrices downloaded from the two databases were integrated and analyzed. Screening of DEGs was performed in the OS versus the control group, followed by Gene Ontology (GO) functional annotation and Kyoto Encyclopedia of Genes and Genomes (KEGG) pathway enrichment analysis. Subsequently, Gene Set Enrichment Analysis (GSEA) and GSVA were performed on the merged data matrix, and a protein-protein interaction (PPI) network was constructed to screen for 10 hub genes. CIBERSORT was applied for the first time, to analyze the infiltration differences of 22 kinds of TIICs in OS and control samples. ROC diagnostic curve and OS survival analyses were performed for 10 hub genes and 22 kinds of TIICs, followed by correlation analysis of hub genes with prognostic value, and 22 kinds of TIICs, was performed. Our research provides potential diagnostic and prognostic biomarkers, and therapeutic targets for OS, and offers new ideas for further study of the molecular mechanisms underlying the development and occurrence of OS. 


\section{Materials And Methods}

\section{Data Mining and Screening for DEGs}

Gene expression profiles and corresponding prognostic information for 88 OS samples were downloaded from a publicly available dataset of OS patients in the TARGET database. The control group consisted of a dataset of gene expression profiles of 396 normal muscle tissue samples, downloaded from the GTEx database. The Affy package [15] of R softwarewas used to perform background correction and data normalization through RMA algorithm. The SVA package [16] was used to merge the CEL files of the two data sets to eliminate the differences between the batches. The effects were visualized by box plots, and the correction between samples was displayed by two-dimensional PCA cluster plots. The Limma package [17] of R softwarewas used to screen for DEGs in OS and control samples. The cutoff value was $\mid \log 2$ fold change $(\log 2 \mathrm{FC}) \mid>1$ at $\mathrm{P}<0.05$. Subsequently, the ggplot2 package [18] was used to draw a volcanic map for visual display.

\section{Gene Ontology Functional Annotation of DEGs and KEGG Pathway Enrichment Analysis, GSEA and GSVA}

GO is an important tool for annotating genes and their products, which facilitates the integration and utilization of biological data [19]. KEGG is a database that integrates the fields of genomics, chemistry, and system function and possesses known biological metabolic signaling pathways [20]. GSEA was used to assess the distribution trend of genes of a pre-defined gene set in a gene table ranked by the degree of correlation with the phenotype, thereby estimating its contribution to the phenotype [21]. The ClusterProfiler package [22] of R software was used to conduct $\mathrm{GO}$ and KEGG enrichment analysis on DEGs. GSEA of the merged gene expression matrix was carried out by selecting c2.cp.kegg.v7.0.symbols.gmt and h.all.v7.0.symbols.gmt as the reference gene set. The cutoff for significant gene enrichment was set at $p<0.05$. To study the differences in the signal enrichment pathway, enrichment of samples from the OS and the control groups was performed. The GSVA package of $R$ software (https://github.com/rcastelo/GSVA), was used to evaluate the role and assign pathway activity conditions. The ggplot2 package was used to draw a map for visual display.

\section{Co-expression analysis, screening of Hub genes and analysis of their functional similarity}

DEGs were imported into STRING (version: 10.5) online software to obtain the relationship between PPIs. STRING (http://www.string-db.org/) is a biological tool for evaluating PPIs [23]. The results were exported in TSV format, and the resulting source files were imported into Cytoscape software [24]. The plug-ins NetworkAnalyzer and CytoHubba [25] were used to screen for Hub genes using the MCC algorithm, and the Hub genes with the top 10 scores were selected. Based on the semantic similarity between the gene 
annotations [26], the R language GOSemSim package [27] was used to calculate the strength of the relationship between molecular function and cell localization among the 10 Hub genes. These genes were sorted according to the average value of functional similarity, with a cutoff value of 0.7 . The results were visualized using the ClusterProfiler package.

\section{Evaluation and Visualization of TIICs}

The gene expression matrix data was uploaded, following pre-processing in CIBERSORT. The samples with an output of $p<0.05$ were filtered to ensure the accuracy of the immune cell population generated by CIBERSORT, and the expression matrices of the 22 kinds of TIICs were obtained [12]. Principal Components Analysis (PCA), one of the most widely used data dimension reduction algorithms, was used to map data to the main feature direction, by obtaining the main projection direction of data, thereby achieving data dimension reduction and de-noising [28]. The filtered TIICS matrix data were subjected to PCA cluster analysis using R software, and a two-dimensional PCA cluster diagram was drawn. The Pheatmap package (https://CRAN.R-project.org/package=pheatmap) was used to draw a heat map to visualize the differences between the 22 kinds of TIICs in OS and control samples. The Corrplot package [29] was used to draw a correlation heat map to visualize the correlation between the 22 kinds of TIICs in OS. A violin plot drawn by the ggplot2 package was used to visualize the difference in the infiltration of the 22 kinds of TIICs in the OS and control groups.

\section{ROC Diagnostic Analysis and OS Survival Analysis}

The receiver operating characteristic (ROC) curve measures the ability of a diagnostic method to recognize disease, and is an important means to determine the diagnostic test standards (that is, the boundary value between normal and abnormal) [30]. To evaluate the diagnostic value of the Hub genes and the 22 kinds of TIICs, the pROC package [31] of the R software was used to generate ROC curves of the Hub genes and the 22 kinds of TIICs. In the ROC curve, an area under the curve (AUC) value greater than 0.8 was retained by TIICs. According to the expression level of the Hub genes and the infiltration level of 22 kinds of TIICs, the OS samples were divided into high and low expression groups and high and low infiltration groups, using R language Survival package (https://github.com/therneau/survival) and Survminer package (https://github.com/kassambara/survminer), for survival analysis and visualization. Results with $p<0.05$ were retained for display.

\section{Correlation analysis between Hub genes with prognostic value and TIICs}

To further evaluate the correlation between Hub genes with prognostic value and the 22 kinds of TIICs, the ggstatsplot package (https://github.com/IndrajeetPatil/ggstatsplot) of R software was used to perform Spearman correlation analysis between five Hub genes of prognostic value and the 22 kinds of 
TIICs. The results were plotted and displayed by the ggplot2 package. Subsequently, the TIICS most related to the Hub genes were screened, and their correlation with the corresponding Hub gene was further demonstrated. The results were visualized using the ggplot2 package.

\section{Results}

\section{Data Pre-processing and Screening of DEGs}

The box plot results (Fig. 1a, b) show the elimination of the inter-batch difference between the two groups of samples. The two-dimensional PCA clustering chart shows the effect before and after correction (Fig. 1c, d). Following correction, the clustering of the two groups of samples was more obvious, indicating that the sample source is reliable. 373 DEGs were obtained, and the results are presented in volcano plots (Fig. 1e).

\section{GO functional annotation of DEGs and KEGG pathway enrichment analysis, GSEA, GSVA}

The GO functional annotation result showed (Fig. 2a) that the DEGs were primarily involved in biological processes like myofibril assembly, actin-myosin filament sliding, and muscle cell development, and their primary molecular function involved actin binding and actin filament binding; the cellular components were abundant primarily in the actin cytoskeleton, contractile fibers, and myofibrils. KEGG pathway enrichment analysis showed (Fig. 2b) that the DEGs were mostly enriched in ECM-receptor interactions, cGMP-PKG signaling pathway, focal adhesion, and calcium signaling pathway. The GSEA results showed that the enriched pathways were primarily involved in the circadian rhythm and oxidative phosphorylation pathway (Fig. 2c), DNA replication and ECM-receptor interaction pathway (Fig. 2d). Among them, the PRKA family genes were involved in the circadian rhythm, the COX family genes were involved in the oxidation phosphorylation pathway, the MCM family genes were involved in DNA replication, and the COL family genes played a key role in the signal transduction process of the ECM-receptor interaction pathway. The GSEA Hallmark pathway enrichment indicated (Fig. 3a) that the DEGs were mainly involved in myogenesis, oxidative phosphorylation, glycolysis, angiogenesis, and KRAS signaling up. The GSVA enrichment results (Fig. 3b) showed that myogenesis, adipogenesis, oxidative phosphorylation, $\mathrm{PI} 3 \mathrm{~K} / \mathrm{AKT} / \mathrm{mTOR}$ signaling, and the p53 pathway were significantly up-regulated, while E2F targets, WNT beta-catenin signaling, TGF beta signaling and glycosylation signaling pathways were significantly inhibited in OS samples compared to controls.

\section{PPI Network Analysis of DEGs and Functional Similarity Analysis of Hub Genes}


To further explore the relationship between DEGs at the protein level, a PPI network based on the interactions of the DEGs was constructed (Fig. 4a). The first 10 genes in the maximum correlation criterion (MCC) namely, TNNI1, MYL1, MYH6, ACTN2, TTN, ACTN3, TNNT3, MYL3, TNNI2, and TNNC2 were selected as the 10 Hub genes (Fig. 4b). Functional similarity analysis showed that TNNI1, TNNC2 and TNNI/2 were the most closely interacting genes. TNN/1 was the only gene with a critical value $>0.7$, and possessed the highest average functional similarity (Fig. 4c).

\section{Immune Cell Infiltration Results}

PCA analysis revealed (Fig. 5a) that the OS and control samples showed a clustering trend. The results of the cluster heat map (Fig. 5b) showed differences in the 22 kinds of TIICs in the OS and control samples. The correlation heat map results showed (Fig. $5 \mathrm{c}$ ) a significant positive correlation between naive $B$ cells and plasma cells, and a positive correlation between resting CD4 memory T cells, and resting NK cells. A significant negative correlation was found between $\mathrm{MO}$ macrophages and naïve $\mathrm{B}$ cells, plasma cells and monocytes. The violin plot showed (Fig. 5d) that the immune cell infiltration in the OS and control samples were comparable. In the OS sample, the infiltration of follicular helper T cells and resting NK cells was relatively more, while that of plasma cells, activated CD4 memory T cells, gamma delta T cells, monocytes, M0 macrophages and resting dendritic cells was relatively less.

\section{ROC Diagnostic Analysis and OS Survival Analysis}

The area under the ROC curve was between 0.5 and 1. In the case when AUC> 0.5 , the closer the AUC was to 1 , the higher was the diagnostic efficiency. The AUC had lower accuracy at $0.5 \sim 0.7$, certain accuracy at $0.7 \sim 0.9$, and higher accuracy at 0.9 or above [30]. The AUC for the ROC curves of the hub genes were as follows: $M Y H 6$ (AUC=0.998), MYL1 (AUC=0.992), TTN (AUC=1.0), MYL3 (AUC=0.935), TNNI1 (AUC=0.997), ACTN3 (AUC=0.991), TNNI2 (AUC=0.999), TNNT3 (AUC=0.998), ACTN2 (AUC=0.998), TNNC2 (AUC=0.997). The AUC tended to be $0.9 \sim 1.0$ (Fig. 6a, b), indicating that the Hub genes had a relatively high diagnostic value. TIICs retained an $A U C>0.8$ (Fig. 6c). Naive B cells (AUC=0.942), plasma cells (AUC $=0.999)$, follicular helper T cells $(A \cup C=0.814)$, resting NK cells $(A U C=0.864)$, monocytes (AUC=0.987), and M0 macrophages (AUC=0.995) also had relatively high diagnostic values. Kaplan-Meier curve showed (Fig. 7) that the up-regulation of TNNI2 expression in OS correlated with good prognosis, while the up-regulation of TTN, ACTN3, TNNC2, and MYL3 expression correlated with poor prognosis.

\section{Correlation analysis between Hub genes with prognostic value and TIICs}

Correlation analysis results showed that ACTN3, TNNI2, TNNC2, MYL3, and TTN were negatively correlated with $\mathrm{M} 0$ macrophage infiltration and positively correlated with plasma cells infiltration. Among 
them, ACTN3, TNNI2, and TNNC2 most closely correlated with M0 macrophages, while MYL3, TTN correlated with plasma cell invasion (Fig. 8 and 9).

\section{Discussion}

OS is the most common primary malignant bone tumor in children and adolescents [1]. Over the past three decades, although great progress has been made in the treatment of osteosarcoma, the overall survival rate of osteosarcoma patients has not improved much $[3,4,10]$. Recent studies have shown that the gene expression level in OS cells and the TIICs in OS TME were closely related to the escape of the OS cells from the immune system, growth and metastasis $[3,9,10]$. Therefore, identifying the DEGs in OS and the types of TIICs in the OS TME, and exploring the relationship between the two are important in understanding the molecular mechanism underlying the development and occurrence of OS. However, due to technical limitations, in order to identify immune cell infiltration, researchers usually label them on a single surface using immunohistochemistry. However, this method is less effective in identifying relevant cell types, and may be misled by other protein markers, leading to a relatively narrow understanding of the immune response [12]. Integrating genomic mapping and the de-convolution algorithm CIBERSORT, can resolve the analysis of immune cell infiltration, and overcome the major drawback of immunohistochemical analysis. Since this is an emerging technology, no literature report currently exists on the use of CIBERSORT in the analysis of the infiltration patterns of TIICs in OS. In this study, we integrated and analyzed the gene chip sequencing data of OS and control samples downloaded from TARGET database and GTEX database respectively, by bioinformatics-related methods, and screened for Hub genes. CIBERSORT was used to re-mine the integrated expression profile data, and the infiltration patterns of 22 kinds of TIICs in OS were analyzed. To analyze the importance of the Hub genes and 22 kinds of TIICs in the diagnosis and prognosis of OS, the correlation between the Hub genes with prognostic value, and the 22 kinds of TIICs, was studied.

We screened a total of 373 DEGs through the comparison of OS and control samples. To understand the pathways and functions that the DEGs in OS samples were involved in, we carried out GO functional annotation and KEGG pathway enrichment analysis. The GO functional annotation results indicated that the DEGs were mainly involved in biological processes like myofibril assembly, actin-myosin filament sliding, muscle cell development, and their primary molecular function involved actin binding, actin filament binding; the cell components were mainly enriched in actin cytoskeleton, contractile fiber, myofibril. Previous studies have shown that actin-myosin filament sliding, actin cytoskeleton, and actin binding were closely related to the occurrence, invasion and metastasis of OS cells [32-35]. KEGG pathway enrichment analysis showed that the DEGs were mainly enriched in ECM-receptor interactions, cGMP-PKG signaling pathway, focal adhesion, and calcium signaling pathway. Huang et al. [36] reported that focal adhesion exerted traction on cells by connecting actin cytoskeleton to the extracellular matrix, which plays an important role in the invasion and metastasis of OS cells. Previous studies have reported that the calcium signaling and CGMP-PKG signaling pathways may be potential therapeutic targets for tumors [37,38]. To validate the KEGG enrichment results and further understand the pathway enrichment of the dataset, we performed GSEA and GSVA on the merged expression profile data. The results of GSEA 
with c2.cp.kegg.v7.0.symbols.gmt as the reference gene set showed that the enrichment pathways were mainly involved in the circadian rhythm, oxidative phosphorylation, DNA replication and ECM-receptor interaction pathway. Previous reports have shown that oxidative phosphorylation [39, 40], circadian rhythm [41], and DNA replication [42] were closely related to the occurrence and progress of OS. Another study has reported that ECM-receptor interaction pathway plays an important role in the occurrence, development, invasion and metastasis of malignant tumors [43]. Among the Hub genes, PRKA family genes were significantly enriched in circadian rhythm, COX family genes in oxidative phosphorylation, MCM family genes in DNA replication, and COL family genes in ECM-receptor interaction signaling pathway. The proteins encode by these genes may be important regulators of signal transduction processes. The results of GSEA Hallmark analysis with h.all.v7.0.symbols.gmt as reference gene set showed that it was mainly enriched in myogenesis, oxidative phosphorylation, glycolysis, angiogenesis and KRAS signaling up. The results of GSVA enrichment showed that there was a significant difference in signal pathway enrichment between the OS and control groups. Compared to the control group, myogenesis, adipogenesis, oxidative phosphorylation, PI3K-AKT-mTOR signaling, p53 pathway was significantly activated in OS samples, while E2F targets, WNT beta-catenin signaling, TGF beta signaling and glycolysis signal pathways were significantly inhibited. Previous studies have shown that PI3K-AKTmTOR signaling [44, 45], adipogenesis [46], WNT beta-catenin signaling [47, 48], TGF beta signaling [49], glycolysis [40], and p53 pathway [50] play an important role in the development and occurrence of OS. Therefore, our analysis results are consistent with previous findings and have high credibility, indicating that these DEGs and signaling pathways may be involved in the occurrence and progression of OS. Myogenesis, calcium signaling pathway, cGMP-PKG signaling pathway, ECM-receptor interaction and KRAS signaling up have not been reported previously in OS research, and are worthy of in-depth discussion.

We also constructed a PPI network of DEGs to detect the interactions between these proteins and screened TNNI1, TNNC2, TNNI2, MYL3, TNNT3, ACTN3, TTN, ACTN2, MYH6, and MYL1 as Hub genes. TNNI1, TNNI2, TNNC2 and TNNT3 are genes involved in encoding troponin [51-54], which plays an important role in the development and occurrence of tumor [55]. ACTN2 and ACTN3 genes encode a-actin [56]. Some studies have reported an interaction between ACTN2, ACTN3 and accessory fibrin tumor suppressor proteins, which are involved in the hypermethylation and inhibition of many oncogenes [57]. The high expression level of ACTN3 may lead to poor prognosis in patients with acute myeloid leukemia [58]. TTN has been proven to be an oncogene [59]. Mutations occur frequently in a variety of tumor types such as lung squamous cell carcinoma, lung adenocarcinoma and colorectal adenocarcinoma [60, 61]. MYL 1, MYL3, and MYH6 are genes encoding myosin [62, 63], and there is evidence of myosin promoting tumorigenesis, migration, and invasion $[64,65]$. However, the above genes have not been reported in OS research, which is worthy of further discussion. We also ranked Hub genes by the semantic similarity of their encoded proteins $[26,66]$, and further assessed the strength of interactions among the hub genes. The results suggested that $T N N / 1, T N N C 2$, and $T N N / 2$ showed the strongest interactions, and that they may play a central role in Hub gene interactions. Previous studies have shown that troponin encoded by TNNI1, TNNI2 and TNNC2 [51, 52,54] is involved in the development and occurrence of tumor [55]. Thus, 
we speculate that these three genes may play a key role in the pathogenesis of OS. However, this needs to be confirmed by further studies. In the analysis, $T N N / 1$ showed the highest functional similarity and is therefore worthy of in-depth study.

To understand the pattern of TIICs infiltration in OS, we used CIBERSORT to transform the integrated expression profile data matrix into 22 kinds of TIICs matrices and analyzed further. The results of PCA analysis showed that there was significant population segregation between OS and control samples, while the cluster heatmap indicated differences in TIICs between the OS and control samples. By analyzing the correlation between TIICs and OS, it was found that naive B cells were significantly positively correlated with plasma cells, and resting CD4 memory T cells were positively correlated with resting NK cells. However, there was a significant negative correlation between $\mathrm{M} 0$ macrophages and naive $B$ cells, plasma cells and monocytes. Krebs et al. [67] found that NK cells played an important role in inducing and polarizing the immune response mediated by $\mathrm{CD} 4^{+} \mathrm{T}$ cells. Shi et al. [68] have shown that the recruitment of monocytes may contribute to the accumulation of 27-hydroxycholesterol $(27-\mathrm{HC})$ in breast cancer, and that the interaction of $27-\mathrm{HC}$ with macrophages further contributes to breast cancer development, which is in line with our analysis results. Our analysis also found that in OS samples, the infiltration of follicular helper T cells, resting NK cells was relatively more, while that of plasma cells, activated CD4 memory T cells, gamma delta T cells, monocytes, M0 macrophages, and resting dendritic cells was relatively less, compared to control samples. Gao et al. [69] found that PD-1/PD-L1 mediates the inhibition of follicular helper T cell (TFH) function in OS, which may lead to the development and occurrence of OS. Conversely, activating the TFH function may be a potential target for tumor immunotherapy $[70,71]$. Some studies have reported that the activation of resting NK cells can promote the apoptosis of OS cells [72,73]. Pahl et al. [74] reported that cetuximab can enhance the killing activity of resting NK cells on OS cells, and may be a new therapeutic modality for the treatment of advanced OS. Regarding gamma delta $T$ cells, the study by Li [75] suggested that gamma delta T cell-based tumor immunotherapy may be a viable treatment for OS patients, especially those with recurrence and metastasis. Wang et al. [76] found that gamma delta T cell immunotherapy, combined with decitabine, could enhance the killing effect of gamma delta T cells on OS cells. Monocytes were found to be related to the prognosis of OS. Previous studies have shown that the lymphocyte / monocyte ratio can be used to predict the overall survival rate of patients with OS [77,78]. Thus, TIICs play an important role in the development, occurrence, treatment and prognosis of OS. However, plasma cells, activated CD4 memory T cells, MO macrophages, and resting dendritic cells have not been reported in OS research and therefore need to be explored further.

Additionally, we performed ROC curve analysis on $10 \mathrm{Hub}$ genes and 22 kinds of TIICs. The results showed that the AUC of 10 Hub genes tended to be $0.9 \sim 1.0$, while the AUC of naive B cells, plasma cells, follicular helper T cells, resting NK cells, monocytes, and M0 macrophages was $>0.8$, thereby showing a high diagnostic value in OS. However, no studies have been reported so far to report these findings. Subsequently, we used OS survival analysis to assess the impact of 10 hub genes and 22 kinds of TIICs on OS prognosis. We found that the high expression level of TNNI2 was associated with good prognosis, 
while the high expression level of TTN, ACTN3, TNNC2 and MYL3 indicated poor prognosis. In addition to TTN and MYL3, previous studies have shown that the expression levels of TNNI2, ACTN3 and TNNC2 were closely related to the development, occurrence, invasion, metastasis and prognosis of tumors [7981], which supports our analytical findings. We analyzed the diagnostic and prognostic value of the Hub genes in OS for the first time, and thus this study may serve as a reference for future studies on OS diagnosis and prognostic biomarkers.

Lastly, we analyzed the correlation between Hub genes with prognostic value and 22 kinds of TIICs. The results showed that $A C T N 3, T N N I 2, T N N C 2, M Y L 3$ and $T T N$ negatively correlated with the infiltration of M0 macrophages, but positively correlated with the infiltration of plasma cells. Among them, ACTN3, $T N N I 2$ and $T N N C 2$ were most related to $\mathrm{MO}$ macrophage infiltration, while $M Y L 3, T T N$ were related to plasma cells infiltration. ACTN3 is involved in the encoding of actin [56]. Macrophages can be divided into three subtypes - M0, M1, and M2. Each subtype has different immune functions, and the cytoskeleton that actin constitutes determines the phenotype, polarity, and function of macrophages $[82,83] . M Y L 3$ is one of the genes encoding myosin [62]. Some studies have shown that myosin is involved in maintaining the tension on immune cell membrane, adhesion and moving, secreting vesicles and assisting phagocytosis [84]. Thus it can be seen that ACTN3, TNNI2, TNNC2, MYL3 and TTN may lead to the occurrence and development of OS by affecting the phenotype and function of M0 macrophages and plasma cells in OS.

However, our study has several limitations. First, the CIBERSORT analysis is based on limited gene data, making it deviate from cellular heterotypic interactions, disease-induced dysregulation, or phenotypic plasticity. Second, since the TARGET database lacks the sample data for the control group, this data is downloaded from GTEx database, which may lead to a certain heterogeneity of data. Although we have carried out quality control, homogenization and standardization of the original data, and removed the inter-batch difference between data sets, a larger sample size and higher quality dataset are required to verify the reliability of the results of this study. Finally, our work is involved the mining and analysis of a previously published datasets. Although some of the previous findings are consistent with our analysis, all the predicted results still need to be confirmed using laboratory data.

In conclusion, we analyzed OS at the gene level and the TIICs involved in OS. 10 hub genes (TNNI1, TNNC2, TNNI2, MYL3, TNNT3, ACTN3, TTN, ACTN2, MYH6 and MYL1) were screened. On comparing the OS and control samples, it was found that the follicular helper T cells, resting NK cells, plasma cells, activated CD4 memory $T$ cells, gamma delta $T$ cells, monocytes, $M 0$ macrophages and resting dendritic cells showed obvious infiltration differences. The 10 Hub genes, naive B cells, plasma cells, follicular helper T cells, resting NK cells, monocytes and MO macrophages may be potential biomarkers for the diagnosis of OS. TNNI2, TTN, ACTN3, TNNCXMYL3 may be potential prognostic biomarkers of OS. ACTN3, TNNI2, TNNC2, MYL3 and TTN were closely related to the infiltration of M0 macrophages and plasma cells. Our research may provide a theoretical basis for further exploring the molecular mechanisms underlying the development and occurrence of OS, and serve as a reference for the development of new diagnostic techniques, prognostic biomarkers and targeted therapeutic drugs for OS.

Page 11/26 


\section{Abbreviations}

OS: Osteosarcoma; TME: Tumor microenvironment; TIICs: Tumor-infiltrating immune cells; DEGs: Differentially expressed genes; GSVA: Gene set variation analysis; TARGET: Therapeutically Applicable Research to Generate Effective Treatments; GTEx: Genotype-Tissue Expression; GO: Gene Ontology; KEGG: Kyoto Encyclopedia of Genes and Genomes; GSEA: Gene Set Enrichment Analysis; PPI: Proteinprotein interaction; PCA: Principal Components Analysis; ROC: Receiver operating characteristic; AUC: Area under the curve; TFH: Follicular helper T cell.

\section{Declarations}

\section{Acknowledgments}

This work has benefited from TARGET and GTEx. We thank the TARGET and GTEx network for its generous sharing large amounts of data. We would like to thank the FigureYa (Xiao Ya Hua Tu) for the figure technology support.

\section{Author Contributions}

QQX and WHY conceived and designed the study. WHY, YJD, and EHR conducted the analysis. WHY and QQX drafted the manuscript. GZZ, ZLW, YJD, and EHR participated in result interpretation. QQX reviewed and edited the manuscript. All authors read and approved the final manuscript.

\section{Funding}

This work was supported by The Key Research \& Development and Transformation Project of Qinghai Province for 2018 (2018-SF-113).

\section{Availability of data and materials}

The datasets used and/or analyzed during the current study are available from the corresponding author upon reasonable request.

\section{Consent for publication}

Not applicable.

\section{Ethics approval and consent to participate}

Not applicable.

\section{Conflict of Interest}

There are no conflicts of interest to be reported. 


\section{References}

1. Kansara M, Teng MW, Smyth MJ, et al. Translational biology of osteosarcoma. Nat Rev Cancer. 2014; 14: 722-735.

2. Isakoff MS, Bielack SS, Meltzer P, et al. Osteosarcoma: Current Treatment and a Collaborative Pathway to Success. J Clin Oncol. 2015; 33: 3029-3127.

3. Dean DC, Shen S, Hornicek FJ, et al. From genomics to metabolomics: emerging metastatic biomarkers in osteosarcoma. Cancer Metast Rev. 2018; 37: 719-731.

4. Wang Z, Wang Z, Li B, et al. Innate Immune Cells: A Potential and Promising Cell Population for Treating Osteosarcoma. Front Immunol. 2019; 10.

5. Grivennikov SI, Greten FR, Karin M. Immunity, Inflammation, and Cancer. Cell. 2010; 140: 883-899.

6. Joyce JA, Pollard JW. Microenvironmental regulation of metastasis. Nat Rev Cancer. 2009; 9: 239252.

7. Quail DF, Joyce JA. Microenvironmental regulation of tumor progression and metastasis. Nat Med. 2013; 19: 1423-1437.

8. Heymann M, Lezot F, Heyman D. The contribution of immune infiltrates and the local microenvironment in the pathogenesis of osteosarcoma. Cell Immunol. 2019; 343.

9. Jones KB, Salah Z, Del Mare S, et al. miRNA Signatures Associate with Pathogenesis and Progression of Osteosarcoma. Cancer Res. 2012; 72: 1865-1877.

10. Scott MC, Temiz NA, Sarver AE, et al. Comparative Transcriptome Analysis Quantifies Immune Cell Transcript Levels, Metastatic Progression, and Survival in Osteosarcoma. Cancer Res. 2018; 78: 326337.

11. Yang X, Zhu S, Li L, et al. Identification of differentially expressed genes and signaling pathways in ovarian cancer by integrated bioinformatics analysis. Oncotargets Ther. 2018; 11: 1457-1474.

12. Newman AM, Liu CL, Green MR, et al. Robust enumeration of cell subsets from tissue expression profiles. Nat Methods. 2015; 12: 453.

13. Haenzelmann S, Castelo R, Guinney J. GSVA: gene set variation analysis for microarray and RNA-Seq data. Bmc Bioinformatics. 2013; 14.

14. Ardlie KG, Deluca DS, Segre AV, et al. The Genotype-Tissue Expression (GTEx) pilot analysis: Multitissue gene regulation in humans. Science. 2015; 348: 648-660.

15. Gautier L, Cope L, Bolstad BM, et al. affy - analysis of Affymetrix GeneChip data at the probe level. Bioinformatics. 2004; 20: 307-315.

16. Parker HS, Leek JT, Favorov AV, et al. Preserving biological heterogeneity with a permuted surrogate variable analysis for genomics batch correction. Bioinformatics. 2014; 30: 2757-2763.

17. Ritchie ME, Phipson B, Wu D, et al. limma powers differential expression analyses for RNAsequencing and microarray studies. Nucleic Acids Res. 2015; 43.

18. Ginestet C. ggplot2: Elegant Graphics for Data Analysis. J R Stat Soc a Stat. 2011; 174: 245. 
19. Harris MA, Clark J, Ireland A, et al. The Gene Ontology (GO) database and informatics resource. Nucleic Acids Res. 2004; 32: D258-D261.

20. Kanehisa M, Goto S. KEGG: kyoto encyclopedia of genes and genomes. Nucleic Acids Res. 2000; 28 : 27-30.

21. Subramanian A, Kuehn H, Gould J, et al. GSEA-P: A desktop application for Gene Set Enrichment Analysis. Bioinformatics. 2007; 23: 3251-3253.

22. Yu G, Wang L, Han Y, et al. Clusterprofiler: an R Package for Comparing Biological Themes Among Gene Clusters. Omics. 2012; 16: 284-287.

23. Szklarczyk D, Gable AL, Lyon D, et al. STRING v11: protein-protein association networks with increased coverage, supporting functional discovery in genome-wide experimental datasets. Nucleic Acids Res. 2019; 47: D607-D613.

24. Smoot ME, Ono K, Ruscheinski J, et al. Cytoscape 2.8: new features for data integration and network visualization. Bioinformatics. 2011; 27: 431-432.

25. Chin $\mathrm{C}$, Chen $\mathrm{S}, \mathrm{Wu} \mathrm{H}$, et al. Cytohubba: identifying hub objects and sub-networks from complex interactome. Bmc Syst Biol. 2014; 84.

26. Sevilla JL, Segura V, Podhorski A, et al. Correlation between gene expression and GO semantic similarity. leee Acm T Comput Bi. 2005; 2: 330-338.

27. Yu G, Li F, Qin Y, et al. GOSemSim: an R package for measuring semantic similarity among GO terms and gene products. Bioinformatics. 2010; 26: 976-978.

28. Sturn A, Quackenbush J, Trajanoski Z. Genesis: cluster analysis of microarray data. Bioinformatics. 2002; 18: 207-208.

29. Friendly M. Corrgrams: Exploratory displays for correlation matrices. Am Stat. 2002; 56: 316-324.

30. Zweig MH, Campbell G. Receiver-operating characteristic (ROC) plots - a fundamental evaluation tool in clinical medicine. Clin Chem. 1993; 39: 561-577.

31. Robin X, Turck N, Hainard A, et al. pROC: an open-source package for R and S plus to analyze and compare ROC curves. Bmc Bioinformatics. 2011; 12.

32. Badique F, Stamov DR, Davidson PM, et al. Directing nuclear deformation on micropillared surfaces by substrate geometry and cytoskeleton organization. Biomaterials. 2013; 34: 2991-3001.

33. Fu Y, Yu W, Cai $\mathrm{H}$, et al. Forecast of actin-binding proteins as the oncotarget in osteosarcoma - a review of mechanism, diagnosis and therapy. Oncotargets Ther. 2018; 11: 1553-1561.

34. Jiu Y, Kumari R, Fenix AM, et al. Myosin-18B Promotes the Assembly of Myosin II Stacks for Maturation of Contractile Actomyosin Bundles. Curr Biol. 2019; 29: 81.

35. Sharili A, Allen S, Smith K, et al. Snail2 promotes osteosarcoma cell motility through remodelling of the actin cytoskeleton and regulates tumor development. Cancer Lett. 2013; 333: 170-179.

36. Huang Z, Barker D, Gibbins JM, et al. Talin is a substrate for SUMOylation in migrating cancer cells. Exp Cell Res. 2018; 370: 417-425. 
37. Wang X, Xiao F, Li Q, et al. Large-scale DNA methylation expression analysis across 12 solid cancers reveals hypermethylation in the calcium-signaling pathway. ONCOTARGET. 2017; 8: 11868-11876.

38. Gong L, Lei $Y$, Tan $X$, et al. Propranolol selectively inhibits cervical cancer cell growth by suppressing the cGMP/PKG pathway. Biomed Pharmacother. 2019; 111: 1243-1248.

39. Dey R, Moraes CT. Lack of oxidative phosphorylation and low mitochondrial membrane potential decrease susceptibility to apoptosis and do not modulate the protective effect of $\mathrm{Bcl}-\mathrm{x}(\mathrm{L})$ in osteosarcoma cells. J Biol Chem. 2000; 275: 7087-7094.

40. Zhu R, Li X, Ma Y. miR-23b-3p suppressing PGC1alpha promotes proliferation through reprogramming metabolism in osteosarcoma. Cell Death Dis. 2019; 10: 381.

41. Zhou L, Yu Y, Sun S, et al. Cry 1 Regulates the Clock Gene Network and Promotes Proliferation and Migration Via the Akt/P53/P21 Pathway in Human Osteosarcoma Cells. J Cancer. 2018; 9: 24802491.

42. Goricar K, Kovac V, Jazbec J, et al. Genetic variability of DNA repair mechanisms and glutathione-Stransferase genes influences treatment outcome in osteosarcoma. Cancer Epidemiol. 2015; 39: 182188.

43. van Dijk M, Goransson SA, Stromblad S. Cell to extracellular matrix interactions and their reciprocal nature in cancer. Exp Cell Res. 2013; 319: 1663-1670.

44. Zhang $\mathrm{H}$, Jiang $\mathrm{H}$, Zhang $\mathrm{H}$, et al. Anti-tumor efficacy of phellamurin in osteosarcoma cells: Involvement of the PI3K/AKT/mTOR pathway. Eur J Pharmacol. 2019; 858.

45. Zhang $\mathrm{H}$, Jiang $\mathrm{H}$, Zhang $\mathrm{H}$, et al. Anti-tumor efficacy of phellamurin in osteosarcoma cells: Involvement of the PI3K/AKT/mTOR pathway. Eur J Pharmacol. 2019; 858: 172477.

46. Shimizu T, Ishikawa T, Sugihara E, et al. c-MYC overexpression with loss of Ink4a/Arf transforms bone marrow stromal cells into osteosarcoma accompanied by loss of adipogenesis. Oncogene. 2010; 29: 5687-5699.

47. Cleton-Jansen A, Anninga JK, Briaire-De Bruijn IH, et al. Profiling of high-grade central osteosarcoma and its putative progenitor cells identifies tumourigenic pathways. Brit J Cancer. 2009; 101: 19091918.

48. Danieau G, Morice S, Redini F, et al. New Insights about the Wnt/beta-Catenin Signaling Pathway in Primary Bone Tumors and Their Microenvironment: A Promising Target to Develop Therapeutic Strategies? Int J Mol Sci. 2019; 20.

49. Lamora A, Talbot J, Mullard M, et al. TGF-beta Signaling in Bone Remodeling and Osteosarcoma Progression. J Clin Med. 2016; 5.

50. Wang B, Fang L, Zhao H, et al. MDM2 inhibitor Nutlin-3a suppresses proliferation and promotes apoptosis in osteosarcoma cells. Acta Biochim Biophys Sin (Shanghai). 2012; 44: 685-691.

51. Sheng J, Jin J. TNNI1, TNNI2 and TNNI3: Evolution, regulation, and protein structure-function relationships. Gene. 2016; 576: 385-394. 
52. Gahlmann R, Kedes L. Cloning, structural-analysis, and expression of the human fast twitch skeletalmuscle troponin-c gene. J Biol Chem. 1990; 265: 12520-12528.

53. Wei B, Jin JP. TNNTI, TNNT2, and TNNT3: Isoform genes, regulation, and structure-function relationships. Gene. 2016; 582: 1-13.

54. Takeda S, Yamashita A, Maeda K, et al. Structure of the core domain of human cardiac troponin in the Ca2+-saturated form. Nature. 2003; 424: 35-41.

55. Leung CS, Yeung T, Yip K, et al. Calcium-dependent FAK/CREB/TNNC1 signalling mediates the effect of stromal MFAP5 on ovarian cancer metastatic potential. Nat Commun. 2014; 5.

56. Lek M, Quinlan KGR, North KN. The evolution of skeletal muscle performance: gene duplication and divergence of human sarcomeric alpha-actinins. Bioessays. 2010; 32: 17-25.

57. Agarwal SK, Simonds WF, Marx SJ. The parafibromin tumor suppressor protein interacts with actinbinding proteins actinin-2 and actinin-3. Mol Cancer. 2008; 7.

58. Yang $X$, Pang $Y$, Zhang J, et al. High Expression Levels of ACTN1 and ACTN3 Indicate Unfavorable Prognosis in Acute Myeloid Leukemia. J Cancer. 2019; 10: 4286-4292.

59. Lawrence MS, Stojanov P, Polak P, et al. Mutational heterogeneity in cancer and the search for new cancer-associated genes. Nature. 2013; 499: 214-218.

60. Kim N, Hong Y, Kwon D, et al. Somatic Mutaome Profile in Human Cancer Tissues. Genomics \& Informatics. 2013; 11: 239-244.

61. Greenman C, Stephens P, Smith R, et al. Patterns of somatic mutation in human cancer genomes. Nature. 2007; 446: 153-158.

62. Cohenhaguenauer $\mathrm{O}$, Barton $\mathrm{P}$, Cong NV, et al. Assignment of the human fast skeletal-muscle myosin alkali light-chains gene (MLC1F/MLC3F) to 2q 32.1-2qter. Hum Genet. 1988; 78: 65-70.

63. Granados-Riveron JT, Ghosh TK, Pope M, et al. alpha-Cardiac myosin heavy chain (MYH6) mutations affecting myofibril formation are associated with congenital heart defects. Hum Mol Genet. 2010; 19: 4007-4016.

64. Chuang J, Wang S, Yang W, et al. Sp1 phosphorylation by cyclin-dependent kinase 1/cyclin B1 represses its DNA-binding activity during mitosis in cancer cells. Oncogene. 2012; 31: 4946-4959.

65. Messal HA, Alt S, Ferreira RMM, et al. Tissue curvature and apicobasal mechanical tension imbalance instruct cancer morphogenesis. Nature. 2019; 566: 126.

66. Han JD, Bertin N, Hao T, et al. Evidence for dynamically organized modularity in the yeast proteinprotein interaction network. Nature. 2004; 430: 88-93.

67. Krebs P, Barnes MJ, Lampe K, et al. NK cell-mediated killing of target cells triggers robust antigenspecific T cell-mediated and humoral responses. Blood. 2009; 113: 6593-6602.

68. Shi SZ, Lee EJ, Lin YJ, et al. Recruitment of monocytes and epigenetic silencing of intratumoral CYP7B1 primarily contribute to the accumulation of 27-hydroxycholesterol in breast cancer. Am J Cancer Res. 2019; 9: 2194-2208. 
69. Gao W, Zhou J, Ji B. Evidence of Interleukin 21 Reduction in Osteosarcoma Patients Due to PD-1/PDL1-Mediated Suppression of Follicular Helper T Cell Functionality. Dna Cell Biol. 2017; 36: 794-800.

70. Qiu L, Yu Q, Zhou Y, et al. Functionally impaired follicular helper T cells induce regulatory B cells and CD14(+) human leukocyte antigen-DR- cell differentiation in non-small cell lung cancer. Cancer Sci. 2018; 109: 3751-3761.

71. Hollern DP, Xu N, Thennavan A, et al. B Cells and T Follicular Helper Cells Mediate Response to Checkpoint Inhibitors in High Mutation Burden Mouse Models of Breast Cancer. Cell. 2019; 179: 1191-1206.

72. Nakashima Y, Deie M, Yanada S, et al. Magnetically labeled human natural killer cells, accumulated in vitro by an external magnetic force, are effective against HOS osteosarcoma cells. Int $\mathrm{J}$ Oncol. 2005; 27: 965-971.

73. Buddingh EP, Schilham MW, Eriaty S, et al. Chemotherapy-resistant osteosarcoma is highly susceptible to IL-15-activated allogeneic and autologous NK cells. Cancer Immunol Immun. 2011; 60: 575-586.

74. Pahl JHW, Ruslan SEN, Buddingh EP, et al. Anti-EGFR Antibody Cetuximab Enhances the Cytolytic Activity of Natural Killer Cells toward Osteosarcoma. Clin Cancer Res. 2012; 18: 432-441.

75. Li Z. Potential of human gammadelta T cells for immunotherapy of osteosarcoma. Mol Biol Rep. 2013; 40: 427-437.

76. Wang Z, Wang Z, Li S, et al. Decitabine Enhances Vgamma9Vdelta2 T Cell-Mediated Cytotoxic Effects on Osteosarcoma Cells via the NKG2DL-NKG2D Axis. Front Immunol. 2018; 9: 1239.

77. Liu T, Fang XC, Ding Z, et al. Pre-operative lymphocyte-to-monocyte ratio as a predictor of overall survival in patients suffering from osteosarcoma. Febs Open Bio. 2015; 5: 682-687.

78. Liu B, Huang Y, Sun Y, et al. Prognostic value of inflammation-based scores in patients with osteosarcoma. Sci Rep. 2016; 6: 39862.

79. Yang X, Pang Y, Zhang J, et al. High Expression Levels of ACTN1 and ACTN3 Indicate Unfavorable Prognosis in Acute Myeloid Leukemia. J Cancer. 2019; 10: 4286-4292.

80. Jin Y, Yang Y. Bioinformatics-based discovery of PYGM and TNNC2 as potential biomarkers of head and neck squamous cell carcinoma. Biosci Rep. 2019; 39.

81. Sawaki K, Kanda M, Miwa T, et al. Troponin I2 as a Specific Biomarker for Prediction of Peritoneal Metastasis in Gastric Cancer. Ann Surg Oncol. 2018; 25: 2083-2090.

82. Liu Y, Chen W, Minze LJ, et al. Dissonant response of M0/M2 and M1 bone-marrow-derived macrophages to RhoA pathway interference. Cell Tissue Res. 2016; 366: 707-720.

83. Chen W, Zhao Y, Li XC, et al. Rho-specific Guanine nucleotide exchange factors (Rho-GEFs) inhibition affects macrophage phenotype and disrupts Golgi complex. Int J Biochem Cell B. 2017; 93: 12-24.

84. Maravillas-Montero JL, Santos-Argumedo L. The myosin family: unconventional roles of actindependent molecular motors in immune cells. J Leukocyte Biol. 2012; 91: 35-46. 


\section{Figures}

a

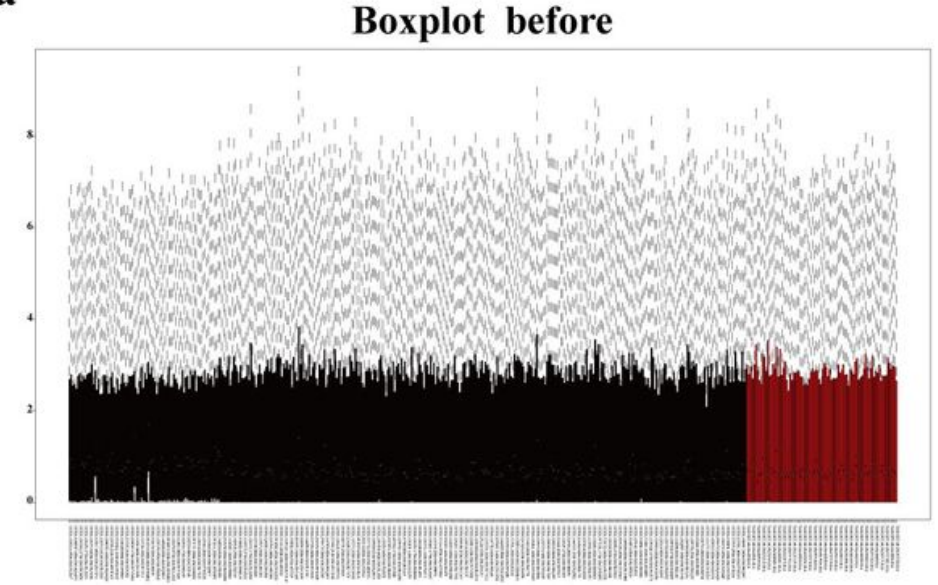

b

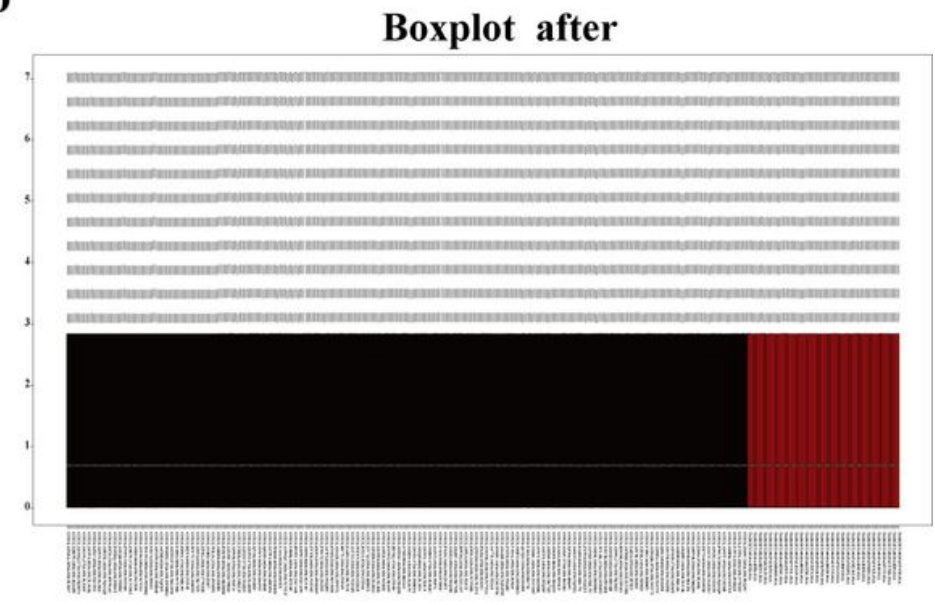

d

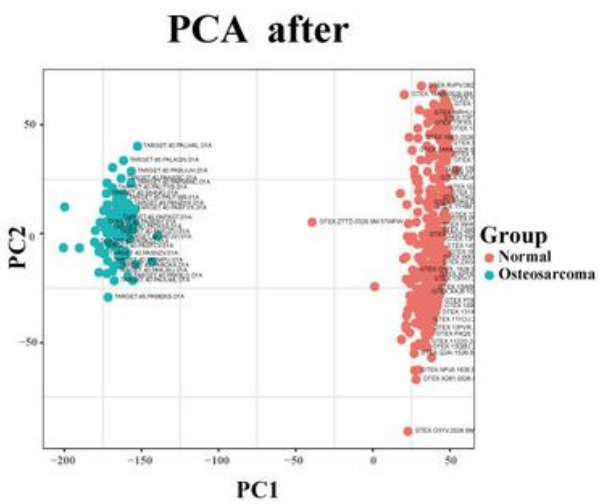

e

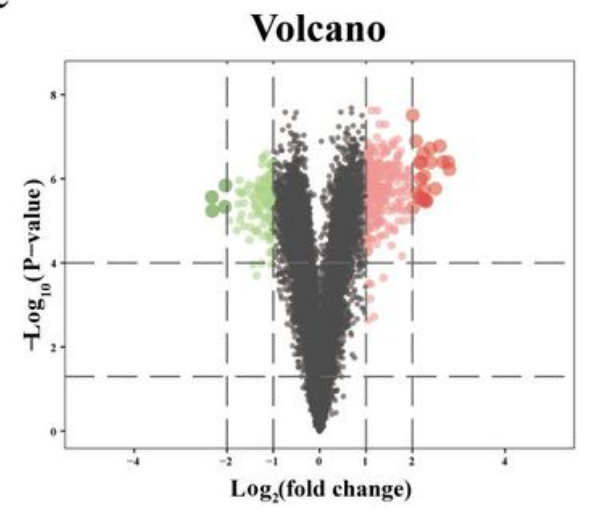

Figure 1

Box plots, two-dimensional PCA plots, and volcano plots of DEGs following data pre-processing. $a, b$ Gene expression matrix data is merged, and boxplots before and after the difference between batches are removed. c, d Two-dimensional PCA clustering graph before and after sample correction. e DEG volcano map. 
a

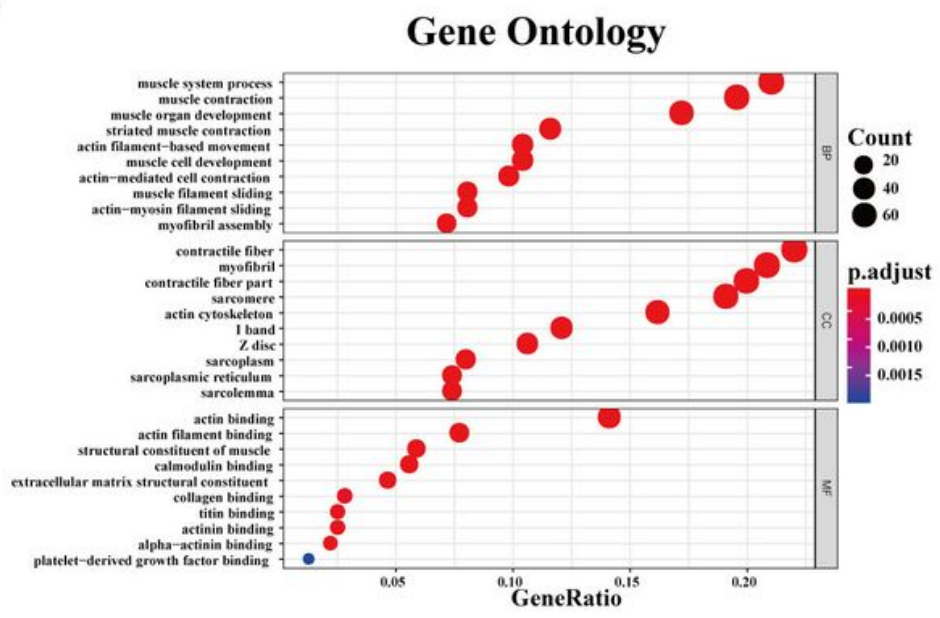

c

GSEA

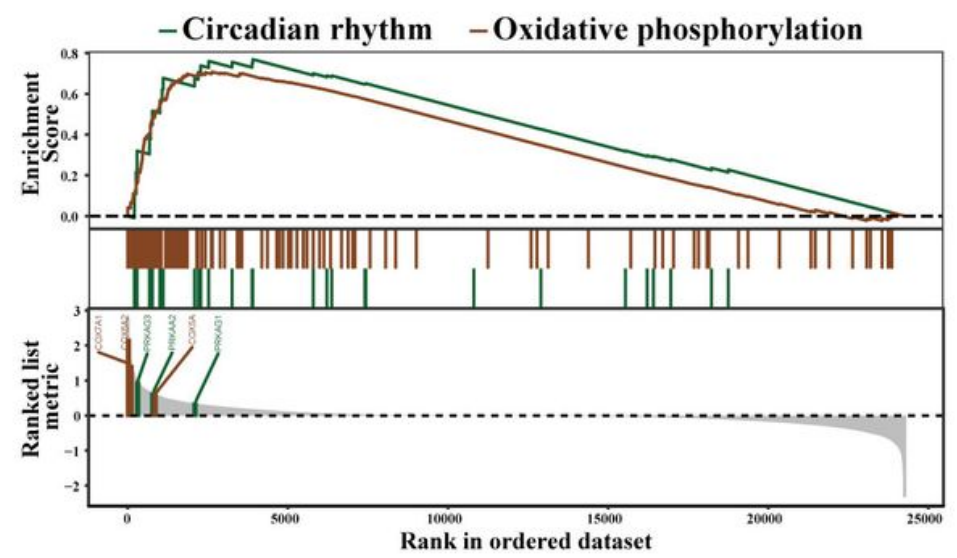

b

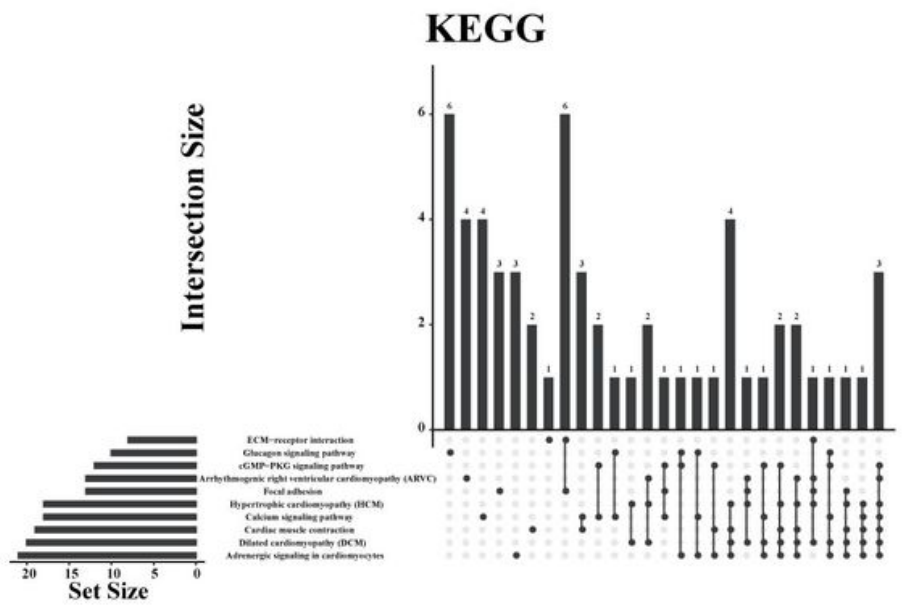

d

GSEA

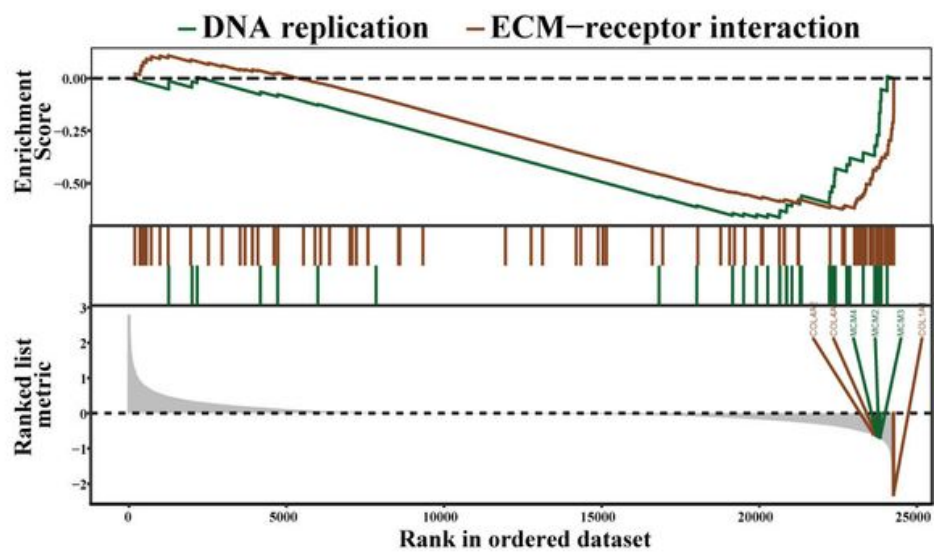

Figure 2

GO analysis and pathway enrichment analysis. a, b GO biological function enrichment analysis and KEGG signal pathway enrichment analysis of DEGs. c, d GSEA. 
GSEA hallmark

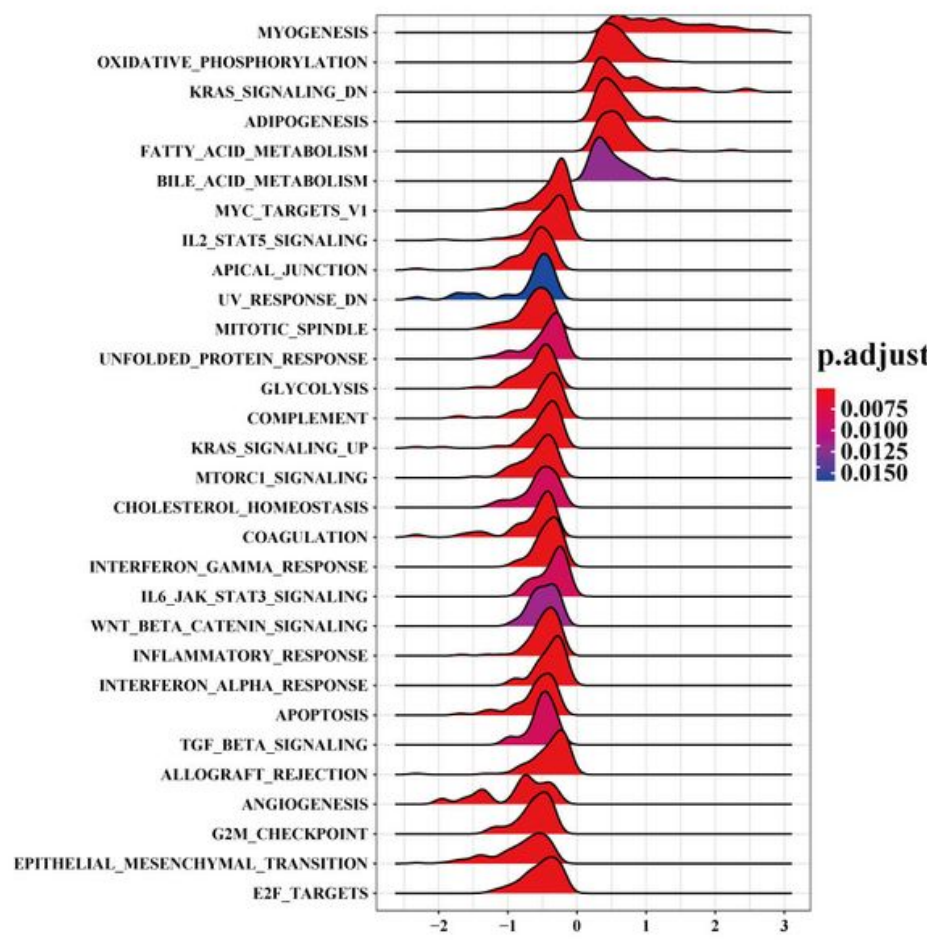

\section{GSVA}

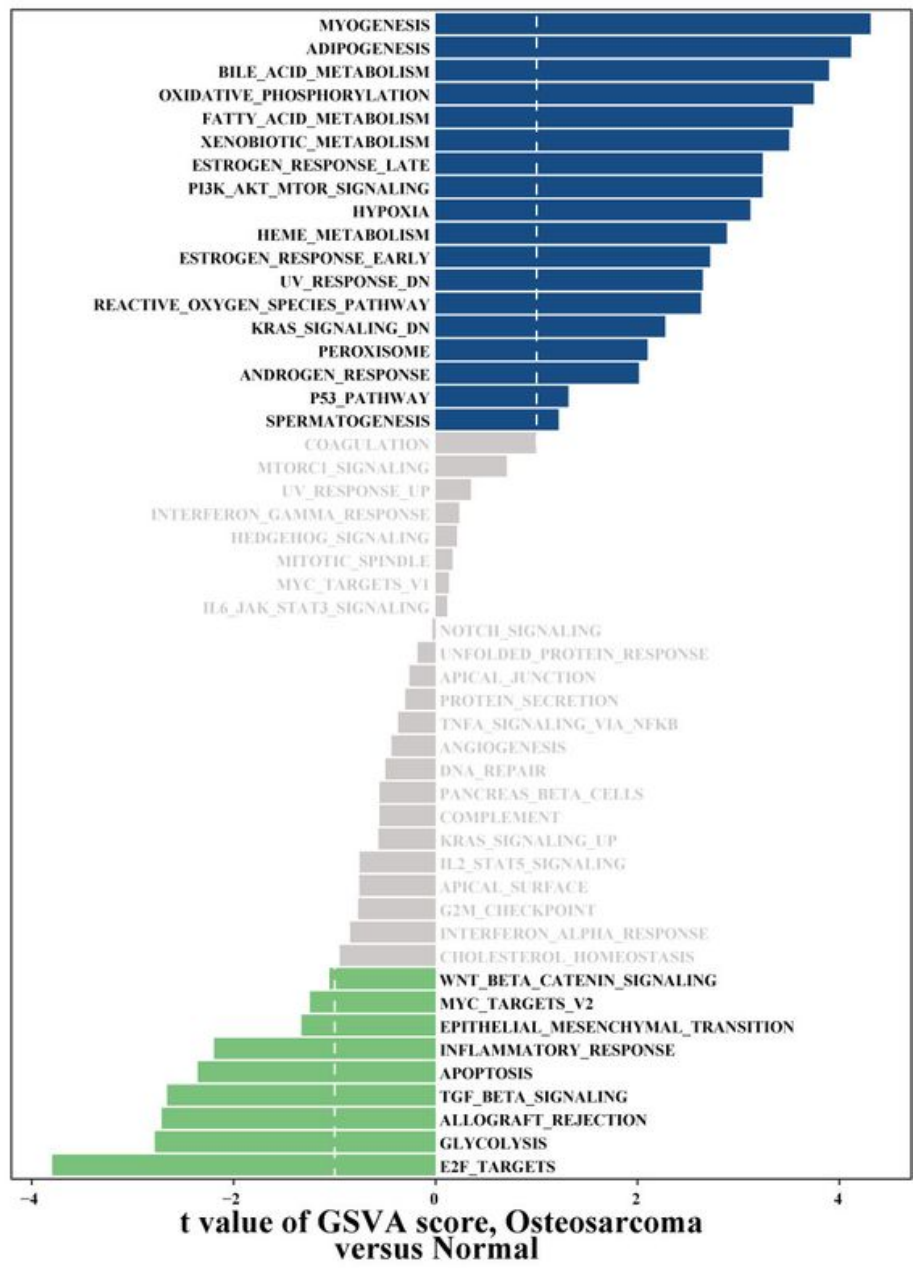

\section{Figure 3}

GSEA, GSVA signaling pathway analysis. a GSEA with signature gene sets. b GSVA scores of OS and control samples. The value of $t$ is shown in the linear model; cutoff value is $|t|>1$. The blue column indicates that the pathway is activated compared to the normal control group, and the green column indicates that it is inhibited. 
b

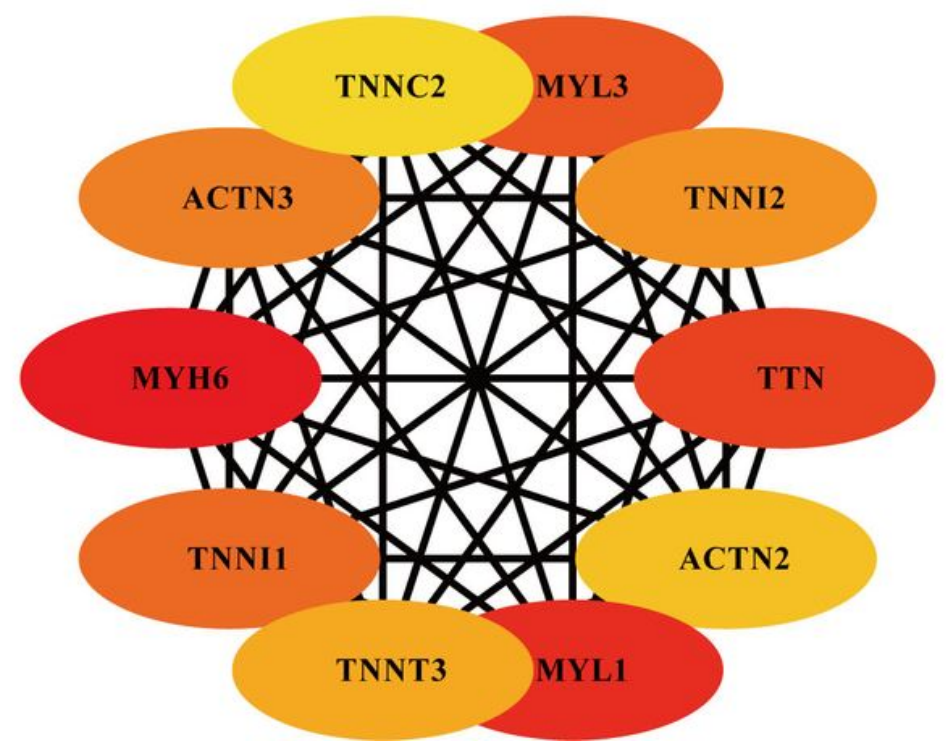

c

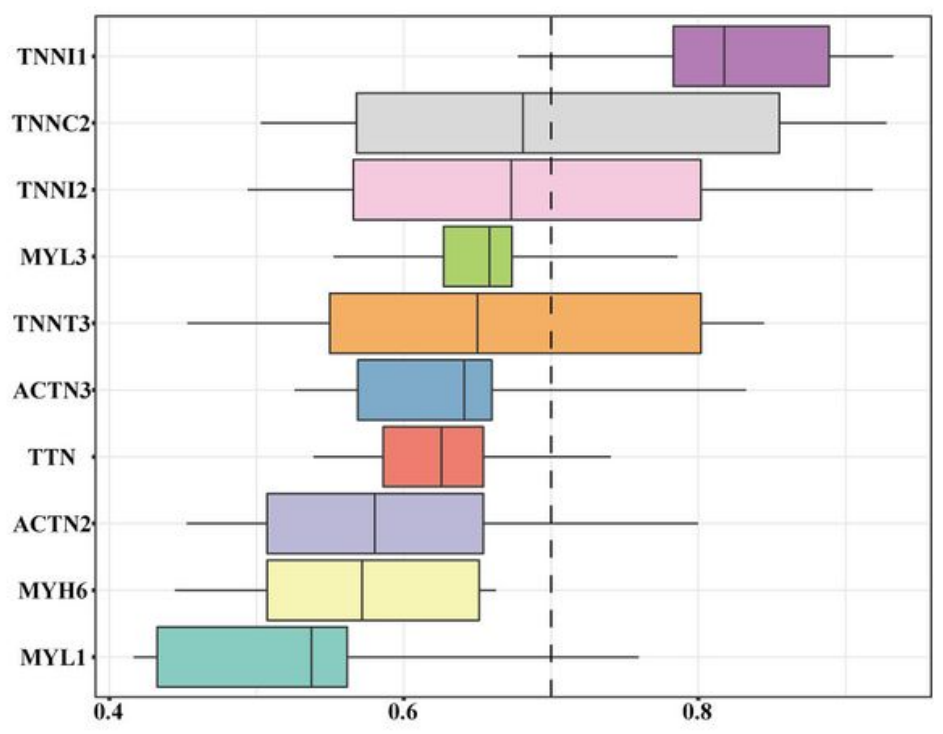

Figure 4

PPI network of DEGs, Hub genes and their functional similarity analysis. a PPI network analysis graph. The node size indicates the clustering coefficient - the larger the node, the larger the clustering coefficient, and the greater the proportion of genes in the network. The node color indicates the degree. The bigger the degree, the more connected the node. Blue represents a large degree, yellow centered, and orange minimum. The thickness of the line represents the overall score. The higher the score, the thicker the line, indicating that the interaction between the two proteins is stronger. b Schematic diagram of the Hub 
gene. The more red the color, the higher the enrichment score. c Functional similarity analysis of hub genes. The functionally similar distributions are summarized as box plots. The box represents $50 \%$ similarity; the upper and lower boundaries show the 75th and 25th percentiles; the lines in the box represent the average of the functional similarity. Genes with a high average functional similarity (cut-off value $>0.7$ ) are considered to be the central genes in the Hub gene interaction; the dashed line indicates the cut-off value.

a

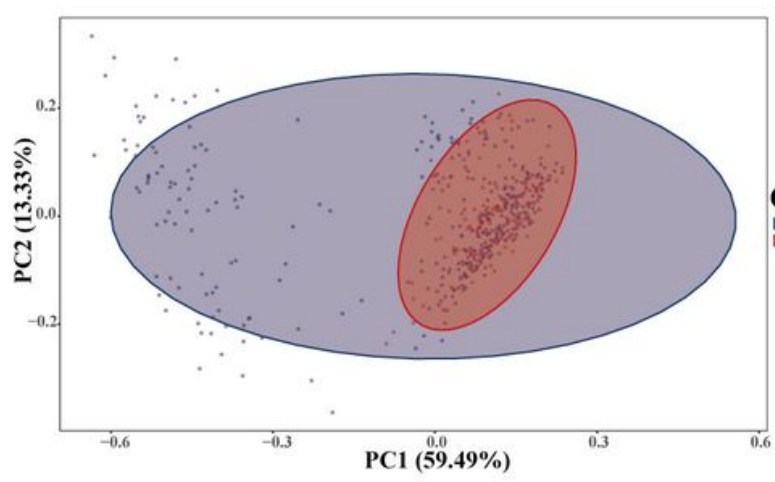

c

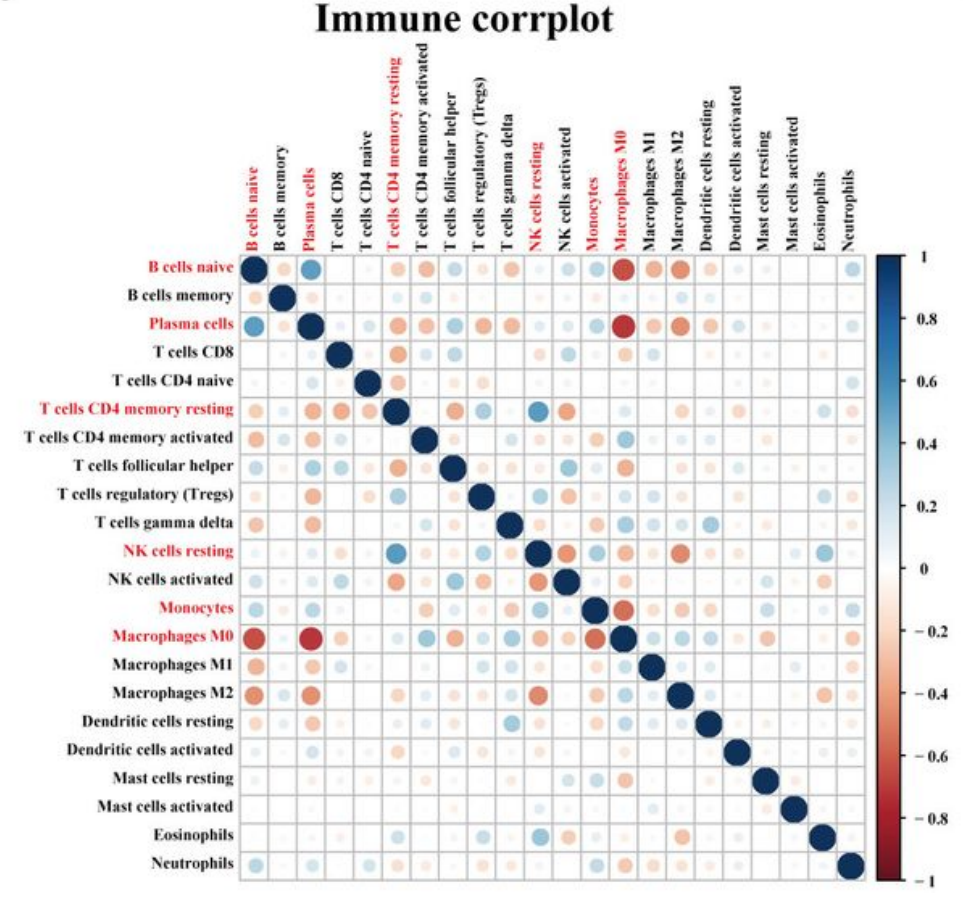

b

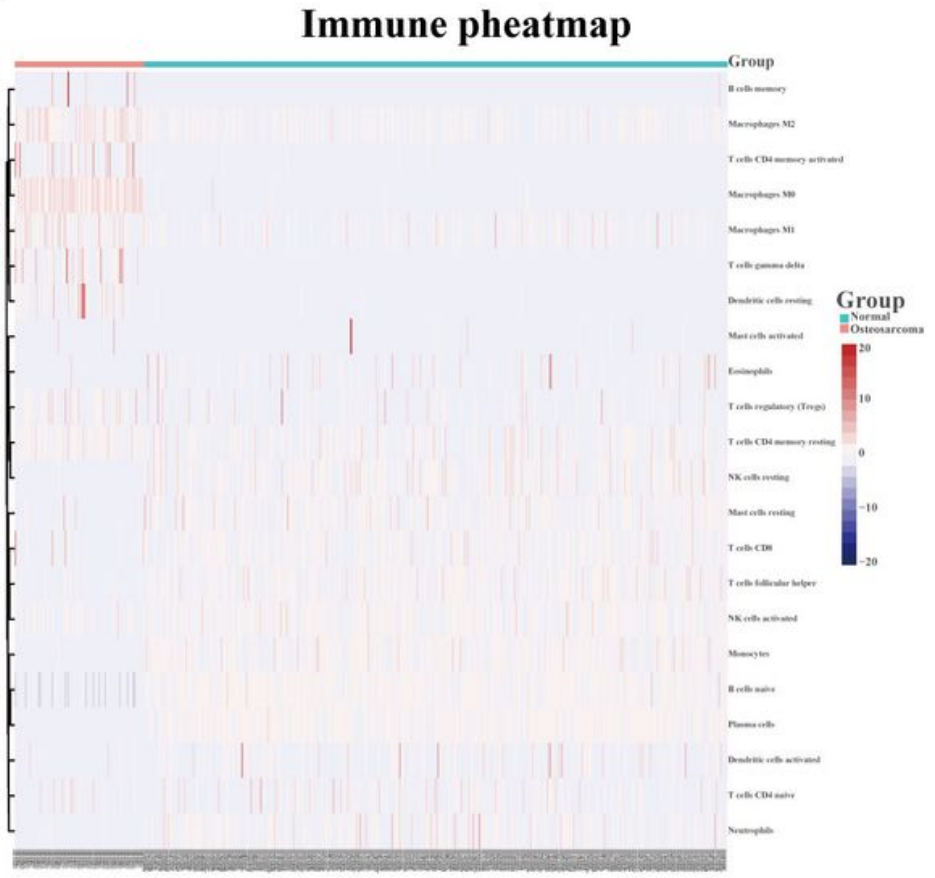

d

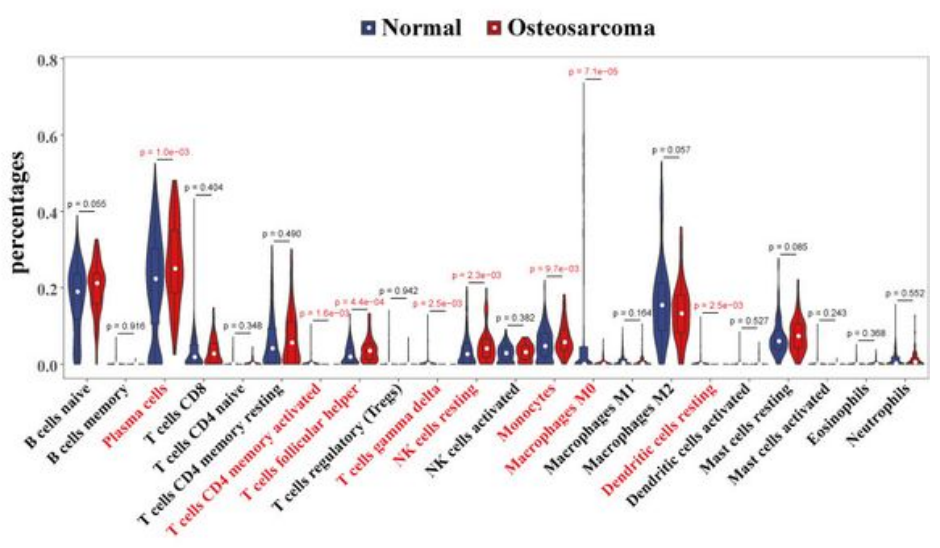

Figure 5 
Evaluation and visualization of TIICs infiltration. a PCA plot of OS samples and control samples. b Heatmap showing the difference in TIICs between OS and control samples. c Correlation heat maps of 22 kinds of TIICs. Blue indicates positive correlation, and red indicates negative correlation. The darker the color, the stronger the correlation. $d$ Violin chart showing the difference in the infiltration ratio of the 22 kinds of TIICs in the OS and control groups. The red mark indicates that there is an infiltration difference between the two groups of samples, $p<0.05$.

a

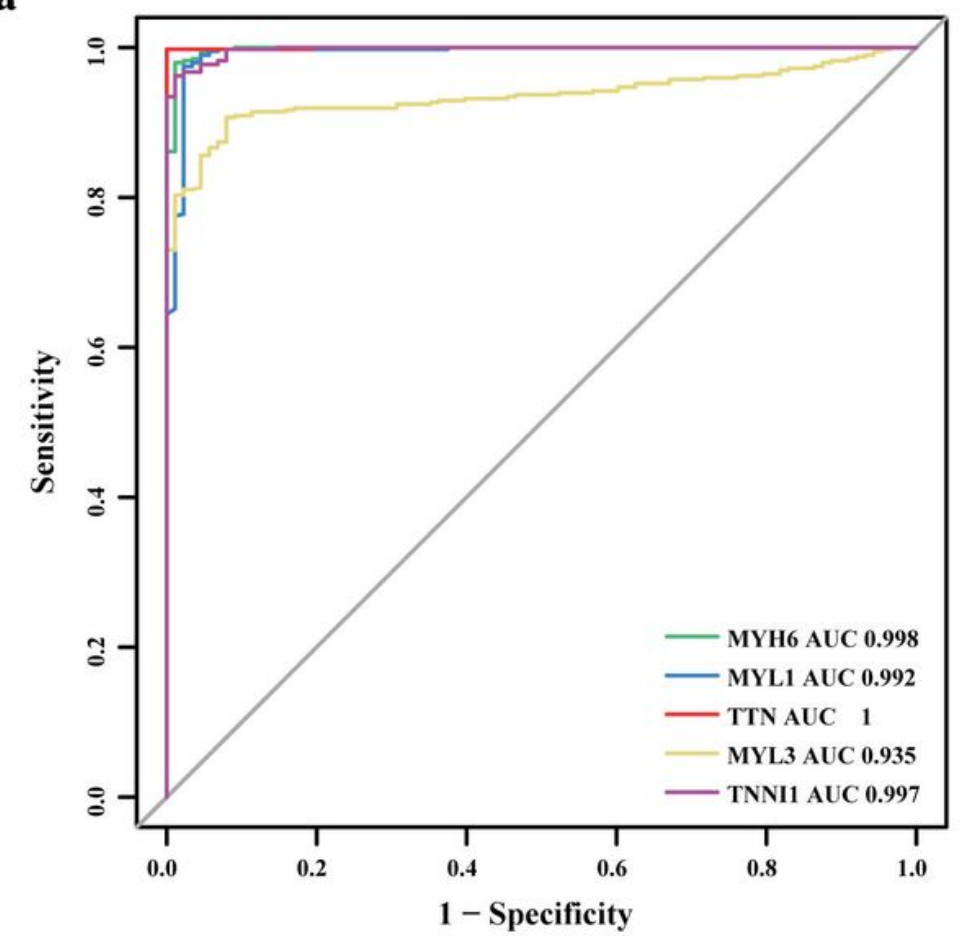

b

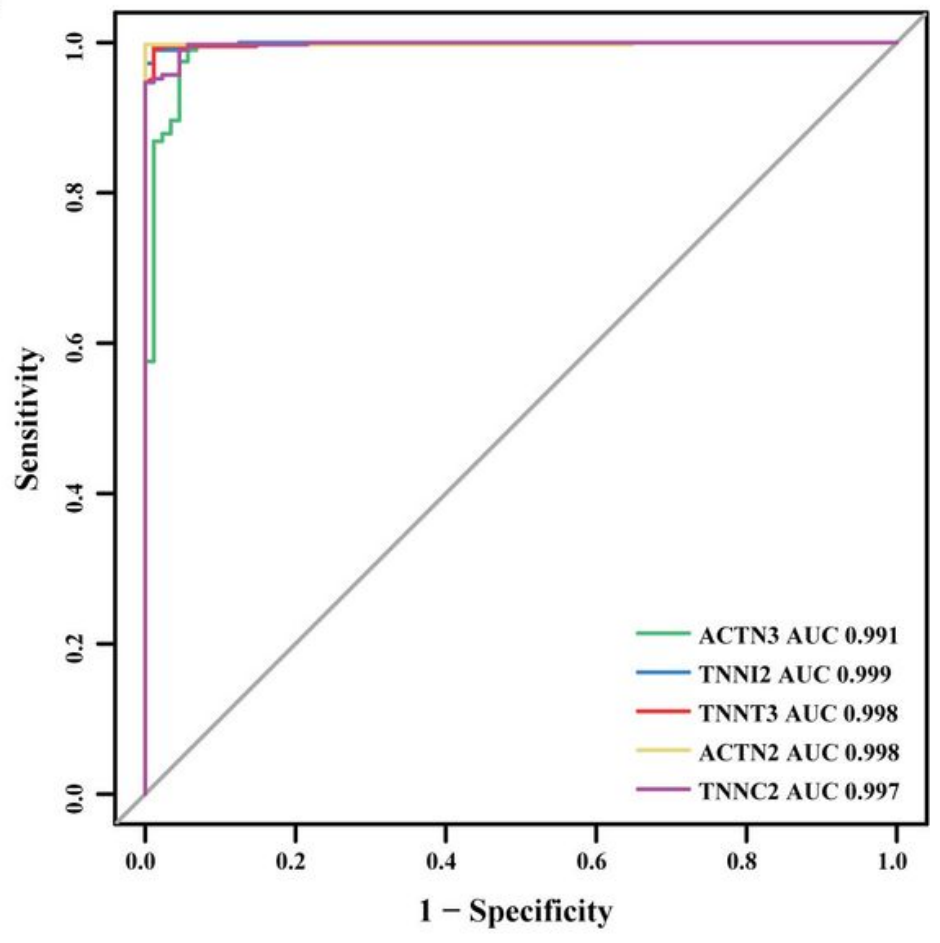

c

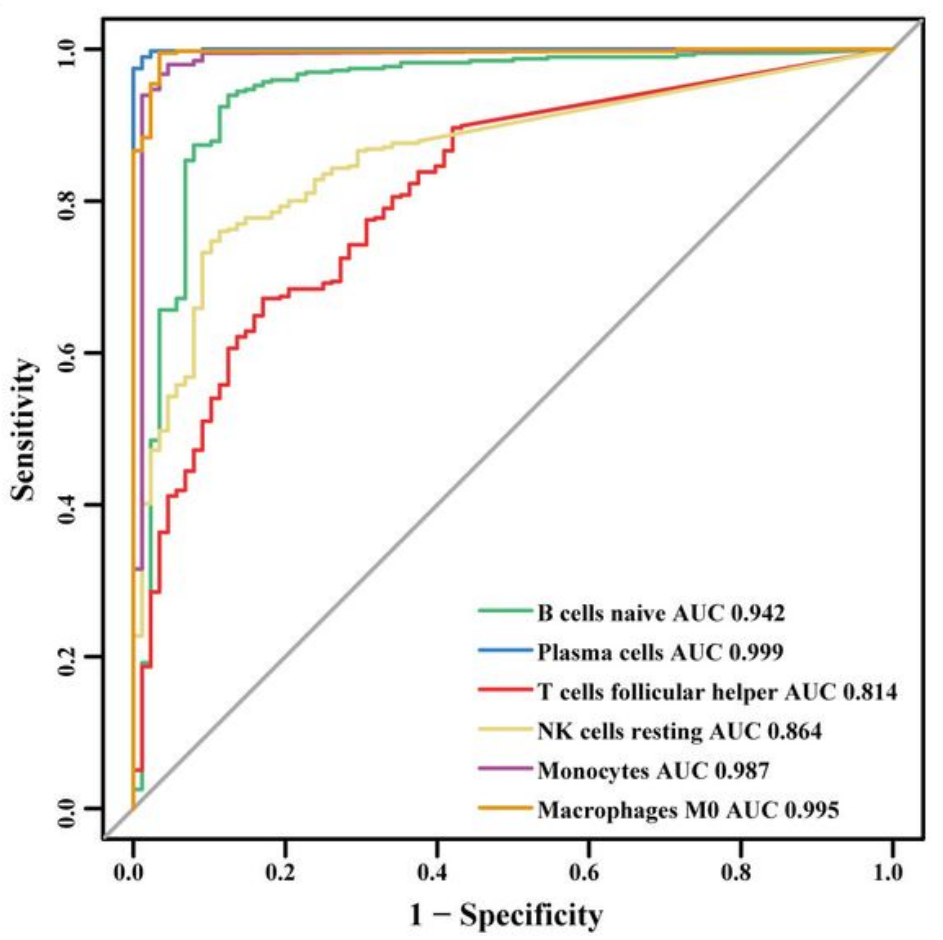

Figure 6 
ROC diagnostic curves. a, b ROC diagnostic curves of 10 hub genes. c ROC diagnostic curve of TIICS.
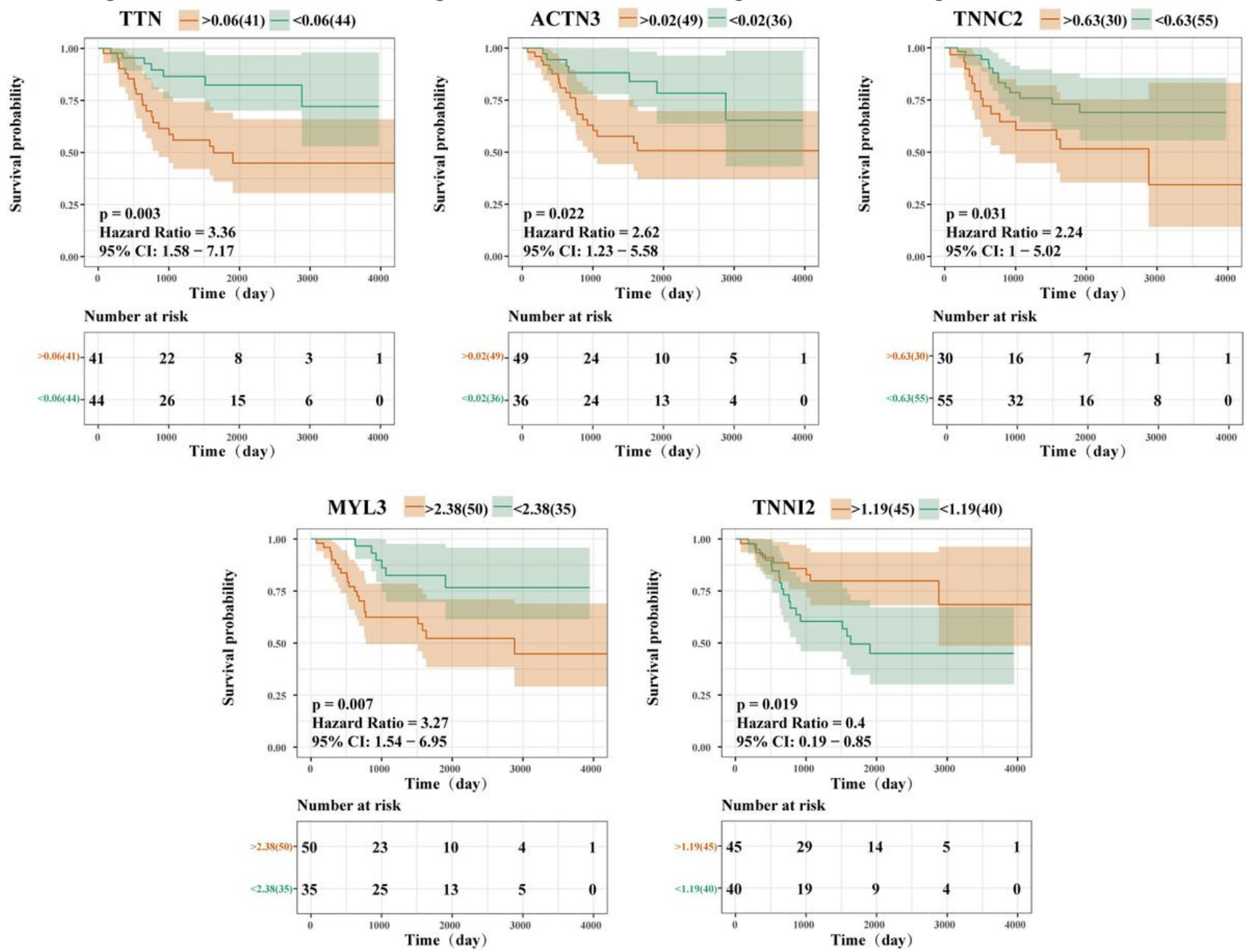

Figure 7

Kaplan - Meier survival curves. The results with $\mathrm{p}<0.05$ are retained for presentation. The horizontal axis represents the total survival time (in days), and the vertical axis represents the survival rate. 

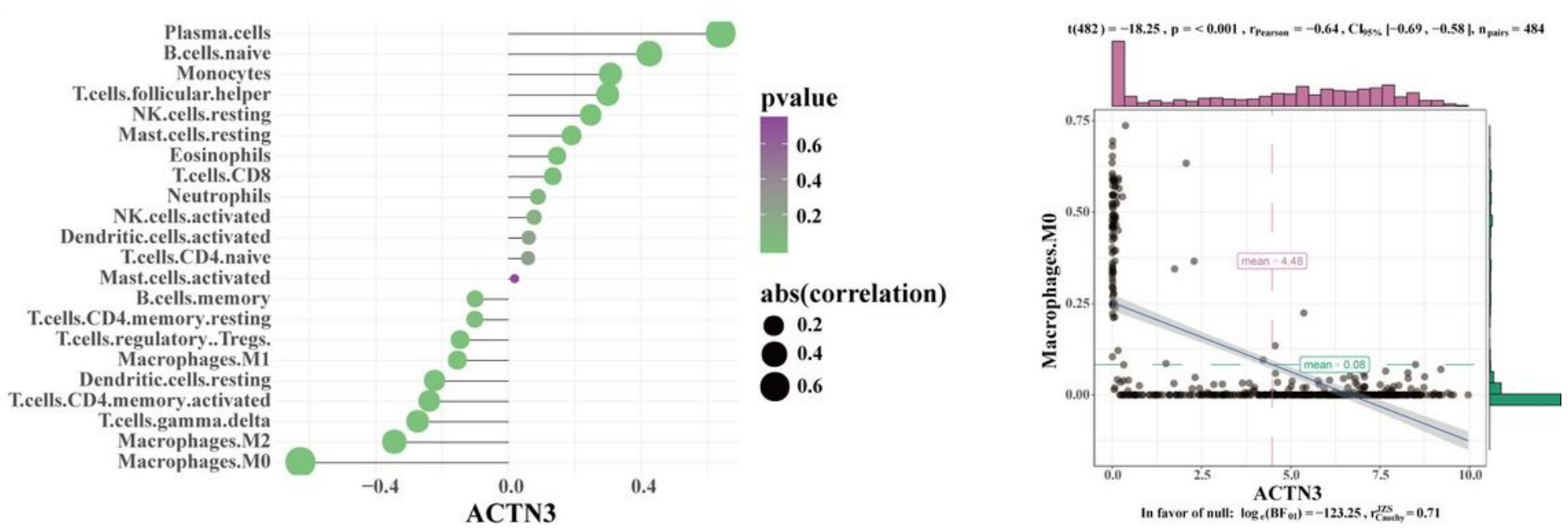

b
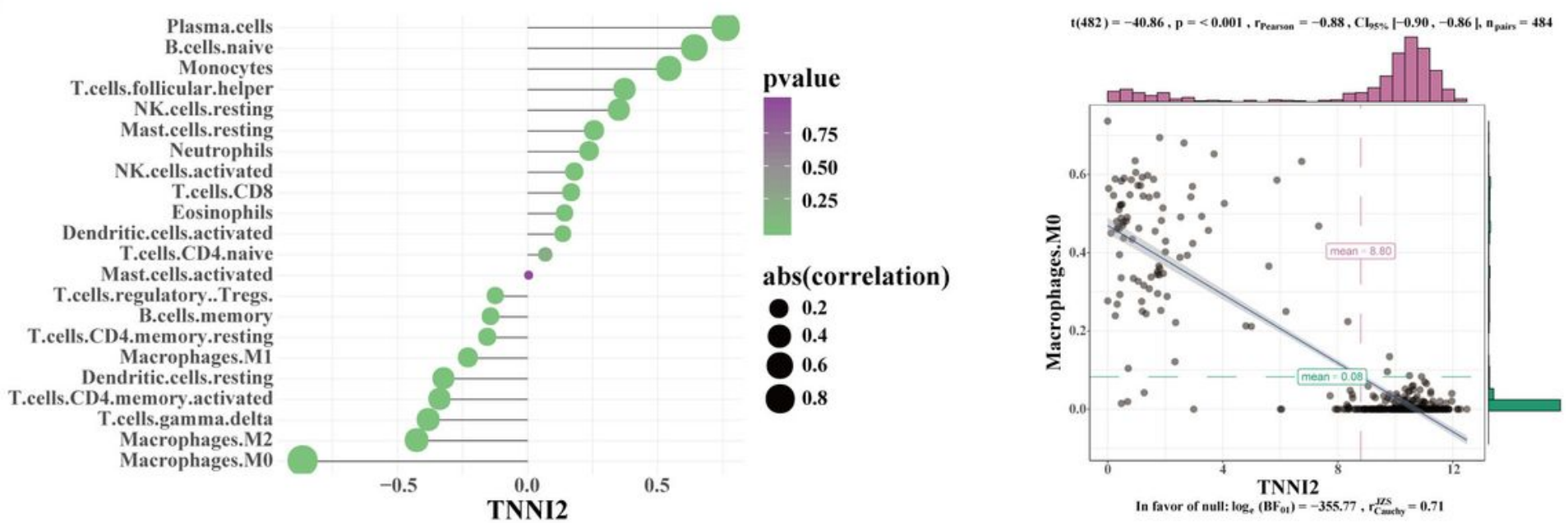

c
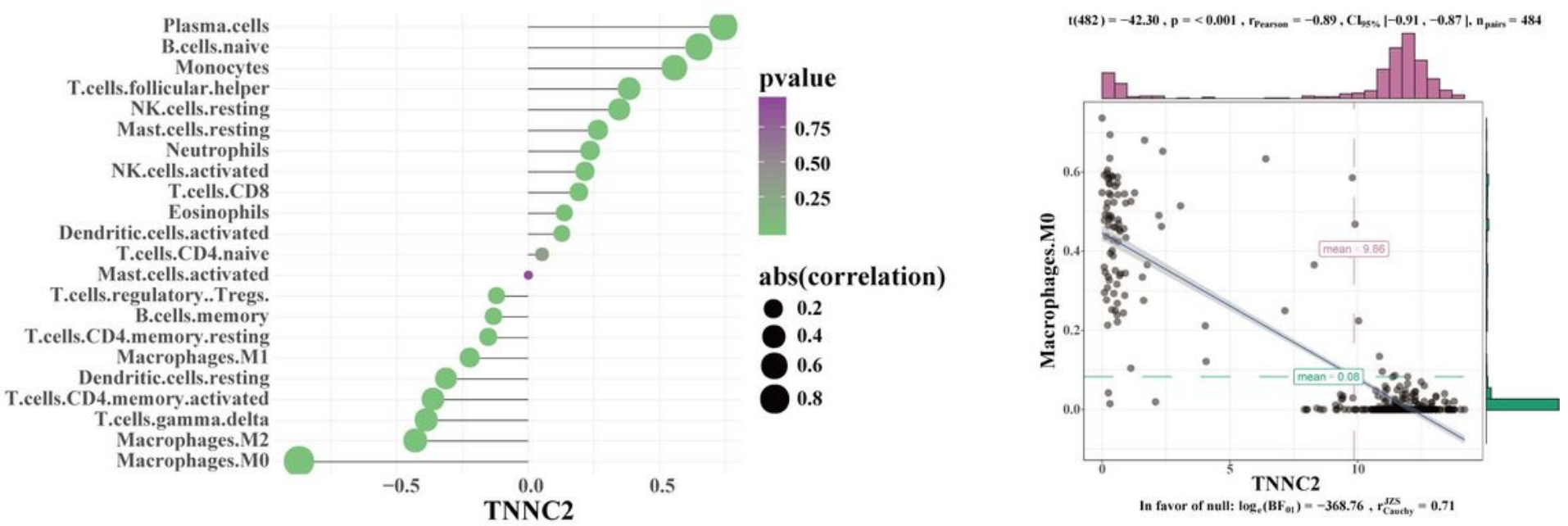

Figure 8

The correlation between ACTN3, TNNI2, TNNC2 and the 22 kinds of TIICs. a, b, c The correlation coefficients of ACTN3, TNNI2 and TNNC2 and the infiltration level of the 22 kinds of TIICS correlate mostly with the infiltration of M0 macrophages. 
a

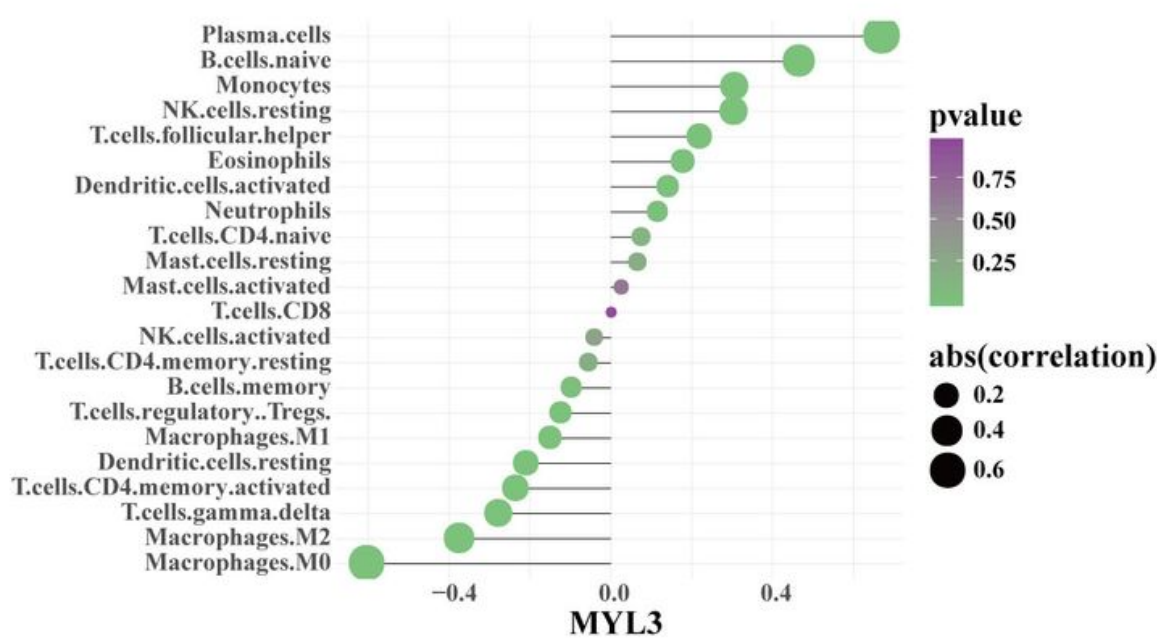

b

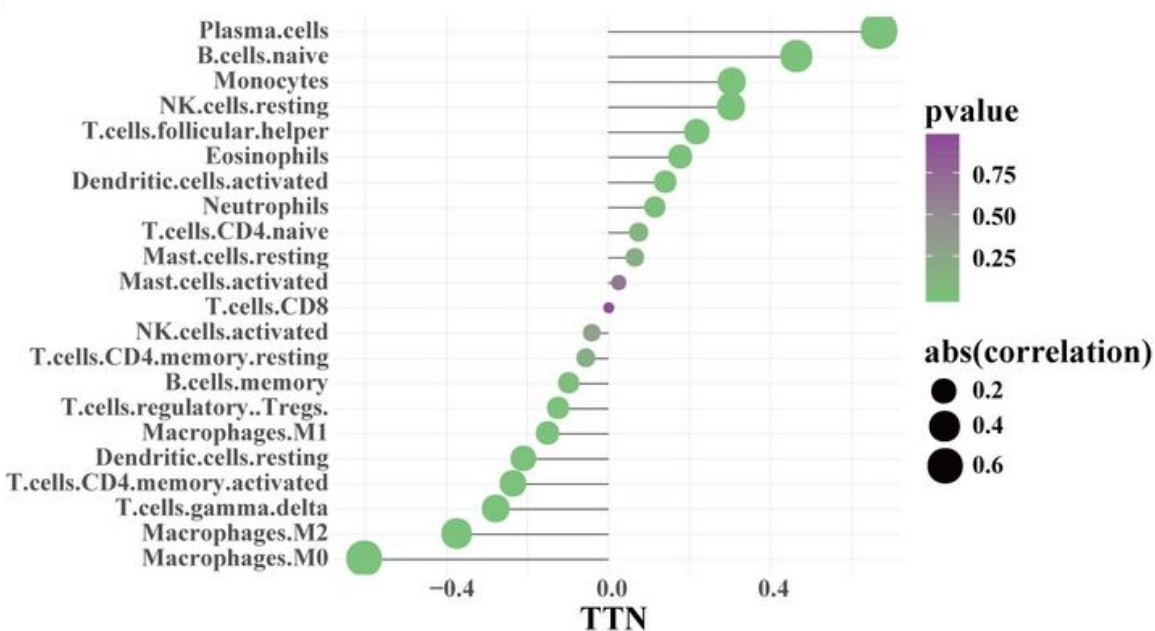

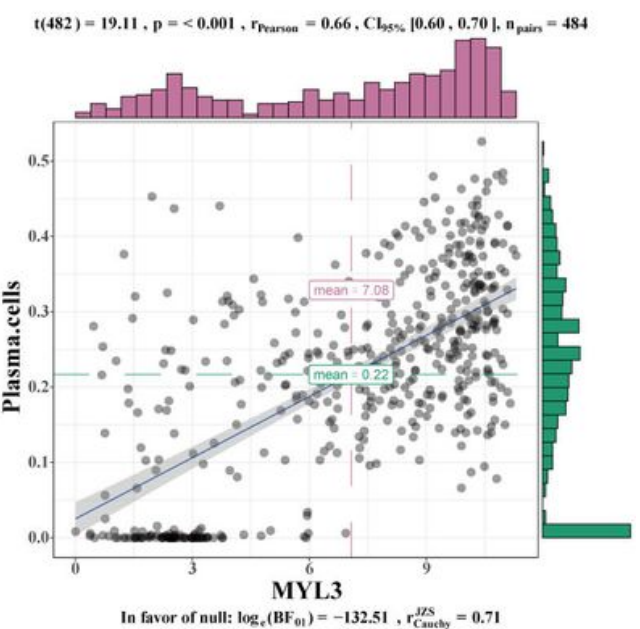

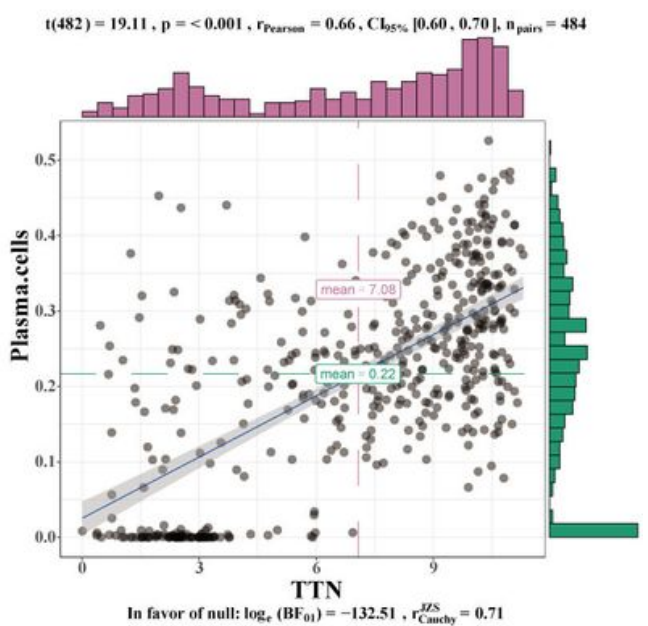

Figure 9

The correlation between MYL3, TTN and 22 kinds of TIICs. a, b The correlation coefficients of MYL3 and TTN and the infiltration level of the 22 kinds of TIICS correlate mostly with the infiltration of plasma cells. 